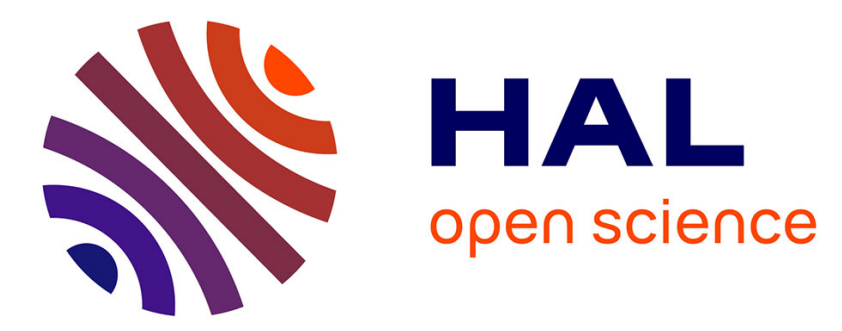

\title{
Chalcogenide glasses as a playground for the application of first-principles molecular dynamics to disordered materials
}

Guido Ori, Assil Bouzid, Évelyne Martin, Carlo Massobrio, Sébastien Le Roux, Mauro Boero

\section{To cite this version:}

Guido Ori, Assil Bouzid, Évelyne Martin, Carlo Massobrio, Sébastien Le Roux, et al.. Chalcogenide glasses as a playground for the application of first-principles molecular dynamics to disordered materials. Solid State Sciences, 2019, 95, pp.105925. 10.1016/j.solidstatesciences.2019.06.014 . hal02349474

\section{HAL Id: hal-02349474 \\ https://hal.science/hal-02349474}

Submitted on 23 Nov 2020

HAL is a multi-disciplinary open access archive for the deposit and dissemination of scientific research documents, whether they are published or not. The documents may come from teaching and research institutions in France or abroad, or from public or private research centers.
L'archive ouverte pluridisciplinaire HAL, est destinée au dépôt et à la diffusion de documents scientifiques de niveau recherche, publiés ou non, émanant des établissements d'enseignement et de recherche français ou étrangers, des laboratoires publics ou privés. 


\title{
Chalcogenide glasses as a playground for the application of first-principles molecular dynamics to disordered materials
}

\author{
Guido Ori ${ }^{1}$, Assil Bouzid ${ }^{2}$, Evelyne Martin ${ }^{3}$, Carlo Massobrio ${ }^{1}$, Sébastien Le Roux ${ }^{1}$, \\ Mauro Boero ${ }^{1}$ \\ ${ }^{1}$ University of Strasbourg, Institut de Physique et Chimie des Materiaux de Strasbourg \\ (IPCMS), CNRS, UMR 7504, 23 rue du Loess, F-67034, Strasbourg, France \\ ${ }^{2}$ Institut de Recherche sur les Céramiques (IRCER), 12 rue Atlantis, F-87068 Limoges, France \\ ${ }^{3}$ University of Lille, CNRS, Centrale Lille, ISEN, UPHF, UMR 8520 IEMN, F-59000 Lille,
}

France

\begin{abstract}
An overview of the major first-principles methods used to simulate condensed phases is presented, with special emphasis on chalcogenide glasses. The scope of this review article is to offer a survey of fundamental algorithms and techniques, accompanied by a few recent examples particularly representative of computational materials science applied to disordered chalcogenide phases. Special attention is devoted to the inclusion of long-range van der Waals dispersion forces, treatment of the exact exchange, dynamical simulations and extraction of optical and dielectric properties. Machine learning techniques are introduced as recent forefront applications of first-principle methods. In this latter case, accurate quantum-mechanics based simulations are crucial to generate a data base exploited by neuronal-network type algorithms to create accurate interatomic potentials (force fields) allowing for large and long-lasting simulations of realistic disordered materials. The atomic-level knowledge provided by the combination of high-performance computing and advanced computational methods pave the route for a rational approach to the design of novel chalcogenides possessing tuned properties for specific applications in next-generation devices.
\end{abstract}

Keywords: molecular dynamics, electronic structure, glass structure, amorphous materials, chalcogenides

\section{Introduction}

Despite remarkable advances in theoretical approaches, numerical algorithms, codes and high-performance computing (HPC) architectures, simulations of solid-state systems large enough to be considered as realistic represent still one of the most 
challenging tasks in computational science. An accurate description of the microscopic properties and the intimate details of the underlying electronic structure call for quantum-mechanical based approaches and, as a consequence, their intrinsic complexity require a many-body methodology, with all the difficulties that this implies.

The main target in this class of atomic-scale simulations is to reproduce, in a realistic way, physical and chemical events occurring in disordered materials as well as in liquid or crystalline systems which undergo a phase transition to a glassy phase [1] This requires something that goes beyond the simple calculation of the atomic and electronic structure of a given set of coordinates $\mathbf{R}_{I}$ representing, for instance, the crystallographic positions of atoms in a solid. Indeed, from a historical standpoint, this idea gave birth to the Molecular Dynamics (MD) field [2-5], whose scope is to replicate, on a computer, systems of interacting particles in a way as close as possible to nature. Dynamical simulations of this type are expected to reproduce the behavior of an extended system over a physical time scale relevant to the properties of interest. Analytic potentials written in terms of a mathematical function $V\left(\mathbf{R}_{I}\right)$ of the atomic coordinates $\mathbf{R}_{I}$ can be very useful in simulating systems for which parameterization, benchmark and assessment on experiments can be done. Yet, this is nearly never the case for a disordered system, especially when electronic structure evolves in time, resulting in cleavage and formation of chemical bonds which are beyond the reach of analytic potentials apart from a very limited class of elements and very specific processes.

Specifically, if a sufficiently accurate force field is available, classical simulations can reproduce all fluctuations and, in a few cases, conformational changes not involving electronic structure modifications. Instead, if electronic structure modifications, chemical reactions including bonds breaking and formation, polarization effects and charge transfer processes are involved, then the use of a quantum approach is unavoidable. Indeed, these phenomena are inherently quantum and can be correctly described only if electrons are explicitly treated in terms of wavefunctions given by the solution of the corresponding Schrödinger or Dirac equations (or one of their many-body generalizations). On the other hand, the computational effort required by a full quantum treatment makes very expensive even the mere optimization of the wavefunctions of the valence electrons for such large and chemically complicated materials

\section{Hartree-Fock based approaches}

The coordinates $\left\{\mathbf{R}_{I}\right\}$ representing the classical Cartesian positions of all the atoms composing a general system are the essential ingredients to start any type of calculation 
and quantum approaches make no exception. To a certain extent, these can be provided by X-ray and neutron scattering experiments for crystalline phases. An amorphous, instead, is much more demanding due to the lack of long-range order. We shall review the basic methods to produce an amorphous system in the forthcoming sections. Yet, for any quantum-mechanics based method, these $\left\{\mathbf{R}_{I}\right\}$ coordinates are not the only variables since the description of the system has to be complemented with the information on the electrons concurring to determine the chemical bonds and the resulting atomic structure.

While nuclei can still be represented as classical degrees of freedom, electrons have to be properly described as quantum objects, hence, in terms of orbitals (wavefunctions) $\psi_{i}$. As a consequence, the Hamiltonian of the system has to contain all the interactions between all the variables involved. The main quantum approaches that have been used to study systems composed of many electrons (and atoms) over the years rely all on some many-body formulation of the fundamental Schrödinger (more rarely Dirac) equation. They are classified according to the basis set or functional form adopted: Hartree-Fock (HF), generalized valence bond (GVB), density functional theory (DFT), configuration interaction (CI), complete active space self consistent field (CASSCF), etc. An excellent and extensive literature is available on these wavefunction based methods [6-8]. A recent comprehensive review can be found in the book from D. Marx and J. Hutter [9], while practical implementations of the various methods are well described in the monography of T. Pang [10].

As routinely pointed out in any textbook, solving the Schrödinger equation for a many-body system is in general a rather demanding task. The first difficulty to face is how to represent electrons with a truly many-body wavefunction $\Psi(\mathbf{q})$, where $\mathbf{q}=\left(\mathbf{x}_{1 \sigma}\right.$, $\left.\mathbf{x}_{2 \sigma}, \ldots, \mathbf{x}_{N \sigma}\right)$ is a multi-dimensional vector defining the position and the spin state $\sigma=$ $\uparrow, \downarrow$ of each one of the $N$ electrons composing the system. Then, one has to solve the associated eigenvalues problem by solving the steady state Schrödinger equation

$$
\hat{H}\left(\mathbf{q}, \mathbf{R}_{I}\right) \Psi(\mathbf{q})=E \Psi(\mathbf{q})
$$

Among all the methods proposed over the years, one of the most popular is the HF approach. Let us remind that this method is based on the variational principle applied in a subspace of wavefunctions where the electronic ground state is assumed to be an antisymmetric combination of single-particle orbitals $\psi_{i \sigma}(\mathbf{x})$, called Slater determinant, on which an orthonormal constraint $\left\langle\psi_{i \sigma} \mid \psi_{j \sigma^{\prime}}\right\rangle=\delta_{i j} \delta_{\sigma \sigma^{\prime}}$ is imposed. In the single particle wavefunction $\psi_{i \sigma}(\mathbf{x})$ the first index $i$ identifies the $i^{\text {th }}$ electron while the second 
one $\sigma$ labels the spin state of each wavefunction, specifically an up arrow for the spin up and a down arrow for the spin down as in any spin-unrestricted formulations. Using this notation, a Slater determinant becomes

$$
\Psi(\mathbf{q})=\frac{1}{\sqrt{N !}} \operatorname{det}\left[\psi_{i \sigma}\left(\mathbf{x}_{j}\right)\right]=\frac{1}{\sqrt{N !}}\left|\begin{array}{cccc}
\psi_{1 \uparrow}\left(\mathbf{x}_{1}\right) & \psi_{1 \downarrow}\left(\mathbf{x}_{1}\right) & \ldots & \psi_{1 \downarrow}\left(\mathbf{x}_{N}\right) \\
\psi_{2 \uparrow}\left(\mathbf{x}_{1}\right) & \psi_{2 \downarrow}\left(\mathbf{x}_{1}\right) & \ldots & \psi_{2 \downarrow}\left(\mathbf{x}_{N}\right) \\
\ldots & \ldots & & \ldots \\
\psi_{N \uparrow}\left(\mathbf{x}_{1}\right) & \psi_{N \downarrow}\left(\mathbf{x}_{1}\right) & \ldots & \psi_{N \downarrow}\left(\mathbf{x}_{N}\right)
\end{array}\right|
$$

The second approximation involved in the HF approach is the analytic form of the Hamiltonian of the system. In fact, the HF version of the many-body Schrödinger equation, written in terms of single-particle orbitals, in atomic units, i.e. assuming as a charge unit the proton (or absolute value of the electron) charge $(e=1)$ and as a mass unit the electron mass $\left(m_{e}=1\right)$, is written as

$$
\left\{-\frac{1}{2} \nabla^{2}+V_{e I}(\mathbf{x})+V_{H}(\mathbf{x})\right\} \psi_{i \sigma}(\mathbf{x})-\sum_{j \sigma^{\prime}} \int d^{3} x^{\prime} \frac{\psi_{j \sigma^{\prime}}^{*}\left(\mathbf{x}^{\prime}\right) \psi_{i \sigma}\left(\mathbf{x}^{\prime}\right)}{\left|\mathbf{x}-\mathbf{x}^{\prime}\right|} \psi_{j \sigma^{\prime}}(\mathbf{x})=\varepsilon_{i} \psi_{i \sigma}(\mathbf{x})
$$

The terms inside the parentheses in the left-hand side of Eq. (3) are the kinetic energy operator, the electron-ion and ion-ion interactions, often referred to as "external Coulomb potential" $\left(V_{\text {ext }}\right)$, and the Coulomb (Hartree) potential acting between two electrons, respectively. We prefer to adopt here the definition $V_{e I}$ (electrons-ions) rather than $V_{\text {ext }}$ because this potential is by no means "external" to the system under study, which is composed of nuclei and electrons. It is just external to the electronic degrees of freedom, but it plays the role of an essential part of the whole interaction responsible for structural and electronic properties of the targeted system. These interactions have the following form

$$
\begin{gathered}
V_{e I}(\mathbf{x})=-\sum_{I} \frac{Z_{I}}{\left|\mathbf{x}-\mathbf{R}_{I}\right|}+\frac{1}{2} \sum_{I, J} \frac{Z_{I} Z_{J}}{\left|\mathbf{R}_{I}-\mathbf{R}_{J}\right|} \\
V_{H}(\mathbf{x})=\sum_{j \sigma^{\prime}} \int d^{3} x^{\prime} \frac{\psi_{j \sigma^{\prime}}^{*}\left(\mathbf{x}^{\prime}\right) \psi_{j \sigma^{\prime}}\left(\mathbf{x}^{\prime}\right)}{\left|\mathbf{x}-\mathbf{x}^{\prime}\right|}=\int d^{3} x^{\prime} \frac{\rho\left(\mathbf{x}^{\prime}\right)}{\left|\mathbf{x}-\mathbf{x}^{\prime}\right|}
\end{gathered}
$$

where the single particle electron density is the result of a sum up on all the spin and particle indexes of the $\psi_{j \sigma},\left(\mathbf{x}^{\prime}\right)$ square modulus wave functions. In practice, this integral form is rarely used, since the Hartree potential $V_{H}$ can be obtained more easily from the solution of the associate Poisson equation

$$
\nabla^{2} V_{H}(\mathbf{x})=4 \pi \rho(\mathbf{x})
$$


thus avoiding the long sum and integration procedures which would be computationally demanding. The second term in Eq. (3),

$$
\sum_{j \sigma^{\prime}} \int d^{3} x^{\prime} \frac{\psi_{j \sigma^{\prime}}^{*}\left(\mathbf{x}^{\prime}\right) \psi_{i \sigma}\left(\mathbf{x}^{\prime}\right)}{\left|\mathbf{x}-\mathbf{x}^{\prime}\right|} \psi_{j \sigma^{\prime}}(\mathbf{x})=\int d^{3} x^{\prime} \frac{\rho\left(\mathbf{x}, \mathbf{x}^{\prime}\right)}{\left|\mathbf{x}-\mathbf{x}^{\prime}\right|} \psi_{i \sigma}\left(\mathbf{x}^{\prime}\right)
$$

is the exchange operator. The name clearly refers to the fact that this operator, acting on the orbital $\psi_{i \sigma}(\mathbf{x})$, exchanges the index $i$ with $j$ and the index $\sigma$ with $\sigma$, and accounts for the fact that from the quantum-mechanics point of view, electrons are indistinguishable particles. Although the exchange is correctly accounted for in HF approaches, it has to be remarked that this term is exactly zero if the ground state wavefunction is not assumed to be antisymmetric. An example of application of this method can be found in the seminal work of Edwards and coworkers [11]. In that study, the pseudo-binary chalcogenide alloy $(\mathrm{GeTe})_{2}\left(\mathrm{Sb}_{2} \mathrm{Te}_{3}\right)$ has been targeted, since this system can be switched electrically in a reversible way from the crystalline to the amorphous phase. This is accompanied by a remarkable change in the conductivity, with clear implications for applications in next-generation electronic devices. HF calculations allowed to compute with appreciable accuracy the electronic structure and to sort out the underlying features responsible for the expected changes in the conductivity properties.

Since neither theoretical nor spectroscopic data were available at the time of this study, this pioneering work has represented a first attempt of using quantum chemical calculations to complement the experimental work by providing pieces of information not directly accessible to experimental probes in the area of chalcogenides. Yet, calculations of the exact exchange term of Eq. (7) were and still are a bottleneck for very large, hence realistic systems. For this reason, in the available literature, HF-based electronic structure calculations on amorphous materials are rare, particularly for chalcogenides, and HF approach are generally used for comparisons [12,13] with computationally cheaper (e.g. density functional theory) methods.

As a word of warning, we recall that the Coulomb potential of Eq. (5) acts only between electron pairs described by single particle wavefunctions. This implies that three-body and higher order terms are neglected and, thus, correlations are not included in HF approaches. These are generally added a posteriori in the so called "post-HF" approaches. In practical applications, the geometry of the model system is optimized at the HF level and then higher order corrections to the energy, coming from perturbation theories are applied. This is, for instance, the case of the widely used Møller-Plesset (MP2) approach [14]. Yet, the total energy of the system is corrected to the second order, but the wavefunctions used to compute the second order perturbation are the ones 
obtained in the uncorrelated HF calculation. As a consequence, wavefunctions and energy functional are not self-consistent and the first derivatives of the MP2 energy functional cannot provide the correct forces needed, for example, to run a molecular dynamics simulation. Nonetheless, HF-based structural optimizations and successive MP2 calculations can be repeated iteratively within a Born-Oppenheimer scheme to sample the potential energy surface [15]. This methodology is in principle very powerful and can lead to rather precise results. However, it has the drawback that the scaling with the system size is very unfavorable. In fact, despite recent attempts of reducing the computational cost [16], the typical scaling of an MP2 procedure is at least $O\left(N^{4}\right)$, whereas the scaling of HF calculations is $O\left(N^{3}\right)$. This implies that only systems with relatively few electrons can actually be treated within these approaches.

For the sake of completeness, we recall that the selection of a specific Hamiltonian and the single-particle expression of the many-body wavefunction do not include all the ingredients needed to actually perform the calculations. One has also to select an appropriate (finite) basis set good enough to approximate the (infinite) Hilbert space spanned by the eigenfunctions of the Hamiltonian of the system and appropriately fit to represent the orbitals $\psi_{i \sigma}(\mathbf{x})$ as linear combinations of analytic functions $\phi_{k}\left(\mathbf{x} ;\left\{\mathbf{R}_{I}\right\}\right)$. In HF approaches, these functions are generally centered on the atomic coordinates $\mathbf{R}_{I}$ of the system, such that

$$
\psi_{i \sigma}(\mathbf{x})=\sum_{k=1}^{M} c_{i \sigma}^{k} \phi_{k}\left(\mathbf{x} ;\left\{\mathbf{R}_{I}\right\}\right)
$$

and the number of analytical functions used, $M$, is also an indicator of the computational cost of the quantum calculation, in the sense that the larger the basis set, the higher the computational workload. Further information can be found in Ref. 17. To date, HF applications to chalcogenide glasses are mostly used as a tool to compute magnetic properties on structural models obtained via less expensive methods and, in particular, to extract susceptibilities and their modifications occurring upon phase transitions [18].

\section{Density functional theory methods}

The density functional theory (DFT), nowadays extensively exploited in a wealth of computer codes and applications, dates back to the early 60s [19-24] and its multidisciplinary impact was worldwide acknowledged by the Nobel Prize in Chemistry in 1998 awarded jointly to Walter Kohn and John A. Pople. Since a rich literature is available $[9,17,25,26]$ we limit our review to the main points with special attention to differences and analogies with the HF methods discussed in the former paragraph. 
Contrary to the HF, DFT gives a reformulation of the many-body quantum mechanics in terms of the electron density, $\rho(\mathbf{x})$, instead of wavefunctions. The scope is nonetheless analogous, providing a theoretically exact description of a (ground state) system composed of interacting electrons, treated quantum mechanically, and point-like nuclei $\left\{\mathbf{R}_{I}\right\}$, treated as classical particles. The total energy functional of such a system can be written in a straightforward way as

$$
E^{\mathrm{KS}}\left[\left\{\psi_{i}\right\}\right]=E_{k}\left[\left\{\psi_{i}\right\}\right]+E_{H}[\rho]+E_{x c}[\rho]+E_{e I}[\rho]+E_{I I}
$$

where the superscript KS stands for Kohn-Sham [20]. The first three terms composing this functional $\left(E_{k}, E_{H}, E_{x c}\right)$ contain the electron-electron interactions, the fourth $\left(E_{e I}\right)$ and fifth one $\left(E_{I I}\right)$ are instead the electron-nucleus and the nucleus-nucleus interactions and they are identical to the ones of equation (4) of the HF formulation. In this case, however, the cumbersome sum on all the wavefunctions is avoided since only the density $\rho(\mathbf{x})$, i.e. a real scalar quantity, is needed to compute this part of the electrostatic interaction. Another analogy with the HF approach is the Hartree term $E_{H}$, namely the Coulomb interaction of equation (5), where, again, only the electron density is needed and, also in this case, the associated Poisson equation simplifies the calculation.

More problematic is instead the calculation of the kinetic functional $E_{k}$. In fact, this term, directly deduced by the Schrödinger equation, would lead to a complicated many-body expression. A dramatic simplification can be obtained by assuming that the electron density can be written in terms of single electron wavefunctions, in perfect analogy with the HF ansatz. The major difference here is that these wavefunctions are just auxiliary mathematical objects, hence not necessarily real molecular orbitals. This ansatz allows writing explicitly $\rho(\mathbf{x})$ as a sum on all the $N^{o c c}$ occupied states

$$
\rho(\mathbf{x})=\sum_{i=1}^{N^{o c c}} f_{i}\left|\psi_{i}(\mathbf{x})\right|^{2}
$$

and only the sum of the square moduli actually matters. The coefficients $f_{i}$ are the occupation numbers, equal to 1 in the case of spin-unrestricted calculations or 2 for doubly occupied spin-restricted cases. The orthonormality constraint

$$
\int \psi_{i}^{*}(\mathbf{x}) \psi_{j}(\mathbf{x}) d^{3} x=\delta_{i j}
$$

is added to ensure the orthonormality of the wavefunctions. With this definition, the kinetic operator reads

$$
E_{k}\left[\left\{\psi_{i}\right\}\right]=\sum_{i=1}^{N^{o c c}} f_{i} \int d^{3} x \psi_{i}^{*}(\mathbf{x})\left(-\frac{1}{2} \nabla^{2}\right) \psi_{i}(\mathbf{x})
$$

and it is diagonal both in the index $i$ and in the argument $\mathbf{x}$ of the wavefunctions, as in a 
non-interacting system of $N^{o c c}$ electrons. The term $E_{x c}[\rho]$ is the new ingredient in the DFT formulation that replaces the exchange operator of equation (7). On one hand, this new functional has to be able to account for the exchange interaction by making use of the electron density (or spin density) only in order to reduce the computational burden. On the other hand, it is more general than the HF exchange, since it is supposed to include also all correlation effects beyond the two-body interaction. The existence of such a functional is somehow the limit of DFT, since the exact analytical expression of this mysterious object is unknown. Nonetheless, sufficiently good approximations are available. For the exchange part only, these approximations, although not being an exact DFT-based formula or a perturbative expansion, are generally deduced within the limit of the homogeneous electron gas [27-29]. By considering only the density $\rho(\mathbf{x})$ given at a specific point $\mathbf{x}$, the so-called local density approximation (LDA) reads

$$
E_{x}[\rho]=-\frac{3}{2}\left(\frac{3}{4 \pi}\right)^{1 / 3} \int d^{3} x[\rho(\mathbf{x})]^{4 / 3}
$$

Analogously, correlation functionals making use of the density $\rho(\mathbf{x})$ have been proposed in the early 80 s [30]. They can be generally written as

$$
E_{c}[\rho]=\int d^{3} x \rho(\mathbf{x}) \cdot \varepsilon_{c}(\rho(\mathbf{x}))
$$

and the explicit form of the integrand term $\varepsilon_{c}(\rho(\mathbf{x}))$ is deduced on the basis of random phase approximation calculation [25]. Semi-local approximations for both the exchange and the correlation (XC) interactions are the ones including the gradient of the density, $\nabla \rho(\mathbf{x})$. These represent the second generation of this class of functionals, having the general expression

$$
E_{x c}[\rho, \nabla \rho]=\int d^{3} x \varepsilon_{x c}(\rho(\mathbf{x}), \nabla \rho(\mathbf{x}))
$$

and making use in practical applications of just the modulus $|\nabla \rho(\mathbf{x})|$ of the gradient, with clear advantages for the practical implementation and the reduction of the computational workload. Despite the fact that these functionals, referred to as generalized gradient corrections (GGA), are somehow arbitrary, they are generally based on rigorous physical and mathematical backgrounds and their accuracy has been carefully assessed over the years [27-38]. The wide and somehow confusing choice of possible GGAs is always a matter of debate and their suitability is often dependent on the nature of the chemical bond they are meant to describe. Among all these alternative formulations, two of the most popular XCs for disordered materials are the PBE [34] and the BLYP [27,36] ones. Their worldwide extensive use is due to the fact that they 
provide the best - or at least the most acceptable - performance in terms of geometrical parameters and relative energies for a wide variety of network-forming materials. As a word of warning, we remind that none of the present XCs includes long-range van der Waals interactions. Their inclusion within DFT approaches will be the focus of the next paragraph. On a general basis, we can safely state that DFT-based calculations have been and are extensively used, even nowadays [39,40], because of their reduced computational cost with respect to HF methods.

\section{Machine Learning schemes and role of first-principle calculations}

A recent forefront application of DFT calculations is the generation of a set of structures $\left\{\mathbf{R}_{I}\right\}$ and corresponding KS total energies $\left\{E_{j}\right\}$ large enough to be exploited as a data base in machine learning (ML) paradigms [41-43] for the construction of precise force fields (FF). Contrary to conventional FFs, which rely on physical considerations, ML potentials employ flexible functional forms to represent reference energies and forces provided by DFT. DFT calculations are then performed on a selected ensemble of points on the potential energy surface (PES). On these bases, ML schemes are designed to extrapolate - or interpolate - the atomic interactions from known selected points accurately computed and located on the PES by previous first-principle optimizations. Consequently, a very close numerical agreement with electronic structure energies and forces can be reached, knowing that the ML potential is by all means more affordable than DFT calculation. Shortcomings of ML potentials are the need for large reference data sets and their limited transferability, which requires a careful validation of the potential.

A generalized neural-network $(\mathrm{NN})$ potential approach for high-dimensional systems of thousands of atoms was introduced by Behler and Parrinello [44]. The high-dimensional NN potentials proposed are based on two key components. The first is the transformation of the atomic positions, (set of Cartesian coordinates) into many-body functions, called atom-centered symmetry functions, describing the local geometric environment of the atoms. This is essential to guarantee that certain properties of the PES are preserved, such as its translational and rotational invariance, and its symmetry with respect to the permutation of atoms of the same type. The second component is a set of atomic NNs. Each of these atomic NNs yields the energy contribution to the total energy as a function of the atomic environment described by the symmetry functions within a given radius. In a nutshell, Fig. 1 shows this procedure and its main steps. Such a ML procedure allowed to construct a reliable and versatile FF for a prototype phase change material, GeTe [41]. Yet, 5000 independent DFT calculations were needed to 
construct the initial data base and this already large number has to be subsequently increased with additional randomly generated structures to cope with different thermodynamic (pressure and temperature) conditions [41]. This preparatory work is indeed a challenging amount of workload even within the computationally affordable DFT framework. As such, it represents the current bottleneck of NN ML schemes.

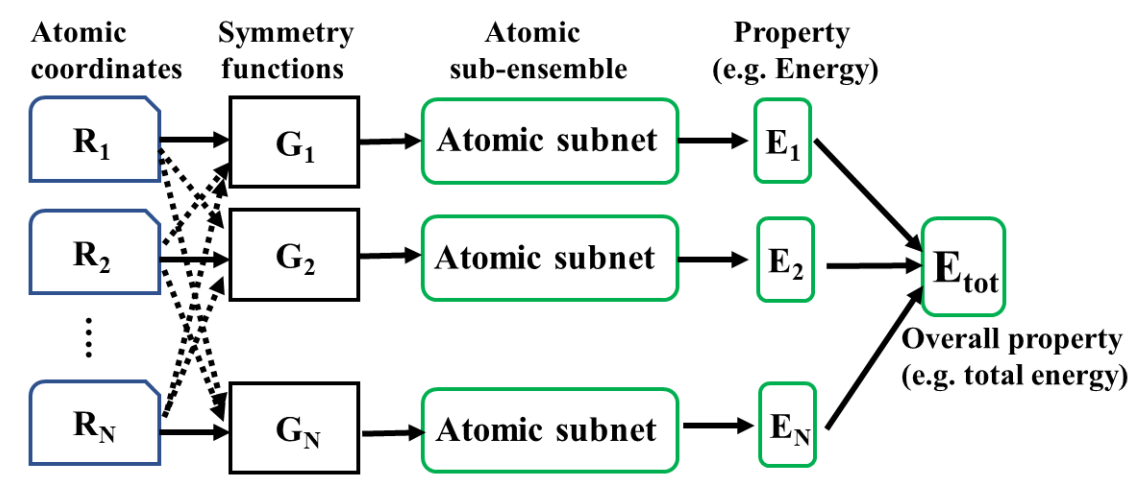

Fig. 1. Schematic representation of a Neural Network architecture and related communication tasks.

Although still subject to ongoing developments, this first attempt at constructing a ML-derived FF for a relatively complicated system has made possible to perform long-lasting molecular dynamics simulations on a nanosecond time scale of very large GeTe nanowires consisting of about 15000 atoms [45], a remarkable achievement still unaffordable a few years ago. On the same line, it is worth mentioning the recent attempt at developing a ML-derived FF for the $\mathrm{Ge}_{2} \mathrm{Sb}_{2} \mathrm{Te}_{5}$ chalcogenide system, prototype for applications in next-generation storage-class memory devices [46,47].

A clear advantage of ML potentials is their intrinsic high degree of parallelization, since the $\mathrm{NN}$ is evaluated independently for each atom or group of atoms and, as such, can exploit a fully parallelism with a relatively reduced amount of communication tasks. In this respect, these schemes are well suited to achieve high-massively parallel computing on thousands of processing elements for large systems treatment. Since the coming exascale machines will have of the order of a billion processing elements, an additional level of parallelism is mandatory, and this is intrinsically granted as summarized in the scheme of Fig. 1. On the other hand, as a word of warning, we wish to point out the fact that obtaining simultaneously energies and their gradients (forces) within a NN approach is nowadays still a stumbling block [41-44].

\section{Complementing DFT: Hybrid functionals and long-range correlations}

Despite the worldwide use of the XC functionals briefly discussed in the previous 
paragraph, cases in which subtle exchange effects come into play and/or long-range correlations become crucial, or even dominant, call for special attention. Although expensive, the first issue can be solved by including in the calculation the exact exchange formulation presented in the discussion of Hartree-Fock approaches. This simple observation is at the basis of any formulation of the so called "hybrid functionals". On a historical perspective, the first one proposed is called B3LYP [48] and, as the acronym suggests, it is a linear combination of the Hartree-Fock exact exchange functional $E_{x} \mathrm{HF}$ with the XC energy $E_{x c}$ in either its LDA or GGA formulation

$$
E_{x c}^{B 3 L Y P}=E_{x c}^{L D A}+a_{0}\left(E_{x}^{H F}-E_{x}^{L D A}\right)+a_{1}\left(E_{x}^{G G A}-E_{x}^{L D A}\right)+a_{2}\left(E_{c}^{G G A}-E_{c}^{L D A}\right)
$$

Specifically, the Becke exchange [27] and the Lee-Yang-Parr [36] functionals are used to compose the B3LYP. The empirical parameters $a_{0}, a_{1}$ and $a_{2}\left(a_{0}=0.20, a_{1}=0.72\right.$ and $a_{2}=0.81$ for this specific functional) are determined by fitting the computed values of atomization energies, ionization potentials, proton affinities, and total atomic energies to experiments and higher level quantum chemical calculations (CI, Coupled Cluster, etc.) for a set of training molecules. Other popular hybrid functionals are the HSE [49] and PBE0 [50] ones. Here we treat them together because they have a common formulation. Also these functionals rely on the calculation of the exact Hartree-Fock exchange at least at short range $E_{x}{ }^{\mathrm{HF}, \mathrm{SR}}$. More precisely, the Coulomb potential is divided into two terms corresponding to a short (SR) and a long range (LR) interaction

$$
\frac{1}{r}=\frac{1-\operatorname{erf}(\omega r)}{r}+\frac{\operatorname{erf}(\omega r)}{r}
$$

For the HSE functional the parameter $\omega$ is (somehow arbitrarily) fixed to the value 0.2 and a mixing coefficient $a=0.25$ is used to write the functional which takes the form

$$
E_{x c}^{H S E}=a E_{x}^{H F, S R}(\omega)+(1-a) E_{x}^{P B E, S R}(\omega)+E_{x}^{P B E, L R}(\omega)+E_{c}^{P B E}
$$

The PBE0 functional is the one obtained by HSE in the limit $\omega=0$

$$
E_{x c}^{P B E 0}=a E_{x}^{H F}(0)+(1-a) E_{x}^{P B E}(0)+E_{c}^{P B E}
$$

As it can be noticed, just the exchange term is modified, while the correlation is unaffected. An interesting application of this class of hybrid functional and their effect on network forming systems has been presented for the specific case of $\mathrm{Ge}_{2} \mathrm{Sb}_{2} \mathrm{Te}_{5}$, a prototype phase change material (PCM) for optical and memory supports [51]. In that work it was shown that although the structural properties are only slightly affected by the inclusion of the exact exchange, electronic properties such as band gap, charge distribution and degree of ionicity/covalence of the chemical bonds turn out to be 
improved. In fact, as far as it could be checked, energy gap and density of states are closer to the experimental ones than those obtained with a standard (PBE) XC.

Contrary to the improvements in the exchange interaction, which are somehow straightforward by resorting to the HF formulation, long range correlations are more demanding. One has to rely on formulations of the van der Waals (vdW) interactions based on either empirical corrections [52,53] or on first-principle calculations making use of the electronic structure provided by DFT [54-60]. The first class of vdW corrections consists in an analytical expression dependent solely on the atomic coordinates of the type

$$
E_{v d W}^{\text {empirical }}=-S_{6} \cdot \sum_{i, j>i}^{N a t} \frac{C_{6}^{i j}}{r_{i j}^{6}} \cdot f_{\text {damp }}\left(r_{i j}\right)
$$

in which the vdW coefficients $C_{6}$ are computed for each pair of atoms or atomic species present in the system. Since short range correlations are already included in the XC functionals currently used in any DFT scheme, a damping auxiliary function

$$
f_{\text {damp }}\left(r_{i j}\right)=\left[1+e^{-\alpha_{6}\left(r_{i j} / R_{v d W}-1\right)}\right]^{-1}
$$

is included to avoid this double counting. The pre-factor $s_{6}$ introduced by Grimme $[52,53]$ is an additional empirical parameter obtained by fitting on a rather large training set of atoms and molecules and its actual numerical value depends on the XC functional used. Thus, its choice has to be consistent with the selected version of both the exchange and the correlation functionals included in the DFT scheme. Provided that this prescription is fulfilled, its application is straightforward and since it does not depend on the electronic structure, it adds a negligible computational cost to the calculation.

For the specific class of materials to which this article is dedicated, we bring to the attention of the reader one of the most recent applications of such an empirical correction. We refer to a throughout analysis of the performance of different XC functionals combined with the Grimme vdW correction on the amorphous $\mathrm{GeTe}_{4}$ system [61], a prototype system of phase-changing materials. These simulations have shown that for an accurate description of the structural properties the choice of the functional is the first crucial step, but then, irrespective of the specific XC prescription adopted, vdW correlations play a non-negligible role in improving in a systematic way the accuracy in terms of both structure factors and pair distribution functions. A direct comparison with experimental data provided by synchrotron radiation measurements features a better agreement with the outcome of the simulations whenever vdW corrections are included. This stringent argument underscores also the importance of 
vdW interactions, often disregarded in chalcogenide compounds simulations, to get a correct structure. On these bases, a detailed insight into local coordination, site geometries and network structure provide a comprehensive atomic-level view which goes beyond the specific amorphous $\mathrm{GeTe}_{4}$ binary system and extends to ternary compounds having $\mathrm{GeTe}_{4}$ as a subsystem.

Analogous importance of this treatment of the vdW corrections has recently been shown to be crucial in the simulation of the structure of both $\mathrm{GeSe}_{4}$ and $\mathrm{GeS}_{4}[62,63]$. In this case amorphous $\mathrm{GeSe}_{4}$ turns out to be characterized by a higher number of fourfold-coordinated Ge atoms. The major drawback of any empirical vdW correction stems from the fact that it is a sort of parameterized restrain imposed to the system and depending solely on the atomic coordinates. No electronic degrees of freedom are included, thus not allowing for any recalculation of the vdW interaction, especially the $C_{6}$ coefficients, upon electronic structure modifications occurring during the dynamical evolution of the system. Yet, this is of fundamental importance when significant modifications of the chemical bonding (bonds cleavage or formation) occur.

To cope with this issue and to overcome the intrinsic limitations of empirical ad-hoc $\mathrm{vdW}$ corrections, several formulations involving the use of the electron density and extensions of the electrostatic potential [54-56] have been proposed. For the purpose of this paragraph, we shall limit the overview to a particular implementation that, starting from the Kohn-Sham (KS) electronic orbitals, upon a unitary transformation into maximally localized Wannier [64] functions [65,66] (MLWF), allows for a calculation "on the fly" of the vdW interaction [57-59] during a dynamical simulation. These functions, although not eigenstates of the Hamiltonian of the system, can be obtained as a unitary transformation of the KS orbitals with explicit expression

$$
w_{i}(\mathbf{x})=\prod_{p=0}^{\infty} \exp \left(-A^{(p)}\right) \cdot \psi_{i}^{K S}(\mathbf{x})
$$

and by using the initial condition $w_{i}^{(0)}(\mathbf{x})=\psi_{i}^{\mathrm{KS}}(\mathbf{x})$ for the iterative solution at step $p=$ 0 . Among all the possible unitary transformations differing just for a phase factor, one has to look for that specific one able to minimize the spread

$$
\Omega=\sum_{n}\left\lfloor\left\langle w_{n}\left|\mathrm{r}^{2}\right| w_{n}\right\rangle-\left\langle w_{n}|\mathbf{r}| w_{n}\right\rangle^{2}\right\rfloor
$$

and for each Cartesian component of $\mathbf{r}=(x, y, z)$ we get at the first iteration

$$
X_{m n}^{(0)}=\left\langle w_{m}^{(0)}|\exp (-i 2 \pi x / L)| w_{n}^{(0)}\right\rangle
$$

and for the subsequent step $p=1$

$$
X^{(1)}=\exp \left(-A^{(1)}\right) \cdot X^{(0)} \cdot \exp \left(A^{(1)}\right)
$$


being $A^{(1)}$ an anti-Hermitian matrix corresponding to a finite step in the direction of the gradient of $\Omega$. Analogous expressions can be obtained for $Y^{(1)}$ and $Z^{(1)}$ along $y$ and $z$, respectively. The steepest descent procedure then reads

$$
A^{(1)}=\Delta t \cdot \frac{d \Omega}{d A^{(0)}}
$$

and

$$
\frac{d \Omega}{d A_{m n}}=X_{m n}\left(X_{n n}^{*}-X_{m m}^{*}\right)-X_{m n}^{*}\left(X_{m m}-X_{n n}\right)
$$

This useful scheme allows for the definition of the center of the MLWF as

$$
\left\langle r_{\alpha, n}\right\rangle=-\frac{1}{G_{\alpha}} \mathfrak{I} m \log \left\langle w_{n}\left|e^{-i G_{\alpha} \cdot x}\right| w_{n}\right\rangle
$$

where $\alpha=x, y, z$ and $G_{\alpha}=2 \pi / L$, being $L$ the size of the simulation box along the $x$ direction. Analogous definitions hold for the other two Cartesian components. Hence, the electronic information concerning the wavefunctions, in terms of MLWF, can be reduced to just four numbers, the three Cartesian coordinates of the center of the Wannier orbital $\mathbf{r}_{\mathrm{n}}=\left(x_{n}, y_{n}, z_{n}\right)$, hereafter indicated as WFC (Wannier function center), and its spread $S_{n}$. This can replace the more cumbersome (discretized) wavefunction $\psi(x, y, z)$ either on the real space mesh $\left(N_{x} \times N_{y} \times N_{z}\right)$ or in the reciprocal space $\left(G_{x} \times G_{y} \mathbf{x}\right.$ $G_{z}$ ) corresponding to a large number of double-precision floating-point data

$$
\psi_{i}(\mathbf{x})=\left(\begin{array}{c}
\psi_{i}(0,0,0) \\
\psi_{i}(1,0,0) \\
\ldots \\
\ldots \\
\psi_{i}\left(N_{x}, N_{y}, N_{z}\right)
\end{array}\right)+\text { c.c. }
$$

amounting to several Mbytes of memory occupation for each orbital. This compact electronic structure information is extremely useful for the analysis of the nature of chemical bonds in a wealth of systems.

For instance, the analysis of the chemical bonding environment in terms of MLWF centers of an amorphous $\mathrm{Ge}_{2} \mathrm{Se}_{3}$ system [67-70] has revealed that beside the expected tetrahedral configurations in which a Ge atom coordinates four Se atoms, more elaborated ring structures arise, including a number of atoms ranging from four to six and sometimes even more. These structures, in turn, may evolve gradually into chains, generally formed by an identical chemical element, hence resulting in sequences of homopolar bonds as sketched in Fig. 2. In these homopolar bonds, WFCs, as in the 
second panel of Fig. 2, are roughly located toward the middle of the Ge-Ge bonds and are characterized by spreads of the order of magnitude of half of the Ge-Ge bond distance, thus accounting for one would expect for a covalent bond. On the other hand, along a Ge-Se bond, the WFCs turn out to be closer to Se atoms, thus evidencing the higher degree of ionicity of these heteropolar bonds.
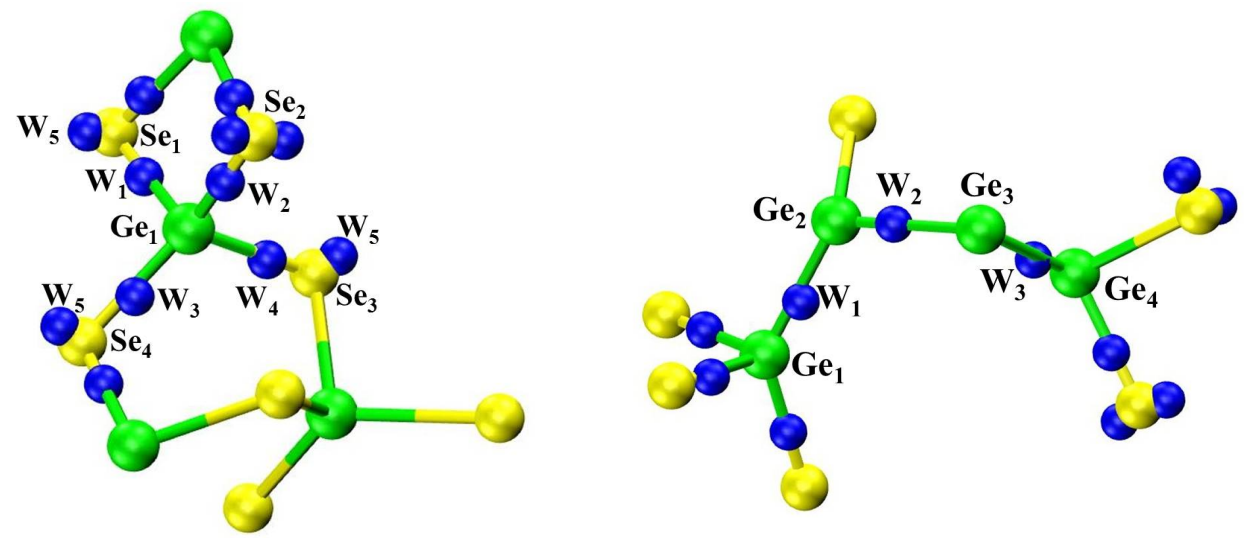

Fig. 2. Details of a dynamically evolving $\mathrm{Ge}_{2} \mathrm{Se}_{3}$ amorphous system. A tetrahedral motif arises in which Ge atoms (green) coordinate four Se atoms (yellow). Moreover, ring structures appear, composed by a number of atoms variable between four and six, as well as chains of atoms of an identical chemical element (homopolar bonds). The blue spheres are WFCs, labeled as $\mathrm{W}_{\mathrm{i}}(\mathrm{i}=1,2, \ldots)$.

Analogously, the peculiar chemical bonding environment of the phase change material $\mathrm{Ge}_{2} \mathrm{Sb}_{2} \mathrm{Te}_{5}$ could be disentangled [71] via a thorough WFCs and spread analysis (see Fig. 3). This provides a versatile and practical way of analyzing the bonding environment of each chemical species participating to the network. The WFCs gathering around an atomic site (Te atoms in this example) indicate a more ionic character of the atomic site, whereas WFCs positioned in the middle of a schematic stick (Fig. 3), as in the case of Sb-Sb chains, underscore the covalent nature of the chemical bond. 


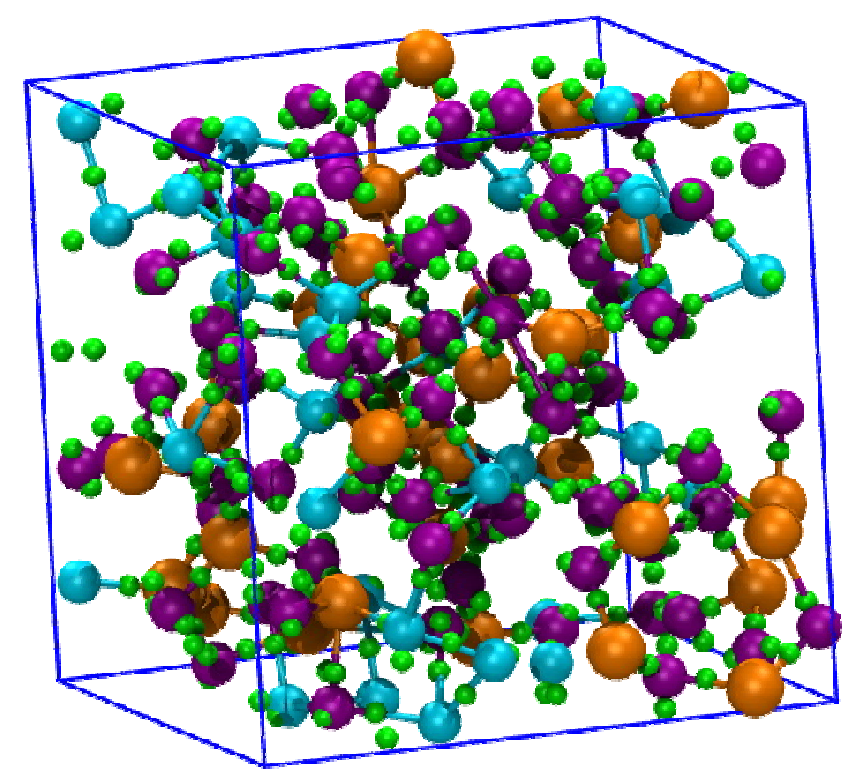

Fig. 3. Phase-change material glassy $\mathrm{Ge}_{2} \mathrm{Sb}_{2} \mathrm{Te}_{5}$ (system size of 144 atoms) with atoms and Wannier centers visualized as spheres. The colors code is orange for Ge, cyan for $\mathrm{Sb}$ atoms, purple for Te, and green for the WFCs.

The joint use of the WFCs, $\mathbf{r}_{n}$ and their spread $S_{n}$ allows for an easy reconstruction of an analytic (localized) wavefunction

$$
w_{n}\left(\left|\mathbf{r}-\mathbf{r}_{n}\right|\right)=\frac{3^{3 / 4}}{\sqrt{\pi} \cdot S_{n}^{3 / 2}} \exp \left(-\frac{\sqrt{3}}{S_{n}}\left|\mathbf{r}-\mathbf{r}_{n}\right|\right)
$$

and this can be used to simplify greatly the Langreth formulation [54] for the $\mathrm{C}_{6}$ coefficient of the vdW interaction. In fact, the original expression

$$
C_{6 n l}=\frac{3}{32 \cdot \pi^{3 / 2}} \int_{|\mathbf{r}| \leq r_{c}} d^{3} r \int_{\left|\mathbf{r}^{\prime}\right| \leq r_{c}^{\prime}} d^{3} r^{\prime} \frac{\sqrt{\rho_{n}(\mathbf{r}) \cdot \rho_{l}\left(\mathbf{r}^{\prime}\right)}}{\sqrt{\rho_{n}(\mathbf{r})}+\sqrt{\rho_{l}\left(\mathbf{r}^{\prime}\right)}}
$$

requires the local electronic densities $\rho_{n}(\mathbf{r})$ within an arbitrary cut-off radius $r_{c}$. Now, the electronic distribution $\rho(\mathbf{r})$ in a DFT framework is a many-body scalar field describing the distribution of all the electrons in the system and not a simple sum of local atom-centered electronic densities. This makes difficult, and to many extents arbitrary, to disentangle separate contributions $\rho_{n}(\mathbf{r})$. Even more cumbersome is the case in which a non-localized basis set (e.g. plane waves) is used. This intrinsic difficulty can be removed by the use of WFCs as in equation (29), which provides the local electron density in terms of square moduli of these analytic wavefunctions as 


$$
C_{6 n l}=\frac{3}{32 \cdot \pi^{3 / 2}} \int_{|\mathbf{r}| \leq r_{c}} d_{c}^{3} \int_{\left|\mathbf{r}^{\prime}\right| \leq r_{c}^{\prime}} d_{c}^{3} \frac{w_{n}(\mathbf{r}) \cdot w_{l}\left(\mathbf{r}^{\prime}\right)}{w_{n}(\mathbf{r})+w_{l}\left(\mathbf{r}^{\prime}\right)}
$$

The expression for the vdW energy does not change and looks similar to equation (35)

$$
E_{v d W}^{W C}=-\sum_{l, n<l} \frac{C_{6}^{n l}}{r_{n l}^{6}} \cdot f_{n l}\left(r_{n l}\right)
$$

Yet, no specific fitting parameter has to be added, contrary to the $s_{6}$ coefficient of Grimme's formulation. Moreover, the damping function turns out to be self-defined by the Wannier functions. In fact, its expression becomes

$$
f_{n l}\left(r_{n l}\right)=\left[1+e^{-\alpha\left(r_{n l} / R_{s}-1\right)}\right]^{-1}
$$

and the cut-off damping radius is simply given by $R_{s}=R_{\mathrm{vdW}}+R_{\mathrm{vdW}}{ }_{\mathrm{vd}}$, namely the sum of the VdW radii of the MLWFs, determined as the radii at which the Wannier function densities $w_{\mathrm{n}}(\mathbf{r})$ become smaller than 0.01. This value is on the verge of the DFT accuracy [24]. Thus, we arrive at the numerical expression

$$
R_{v d W}=\left(1.475-0.866 \cdot \ln S_{n}\right) \cdot S_{n}
$$

in which the MLWF spread $S_{n}$ appears. This is not an arbitrary parameter to be selected by the user, but instead one of the intrinsic results of the unitary transformation of the $\mathrm{KS}$ orbitals. In this way, the $\mathrm{vdW}_{6}$ coefficient is no longer an empirically adjusted constant for each atom type, but a quantity recalculated "on the fly". Therefore, it can become in a straightforward way a time-evolving quantity in first principles molecular dynamics (FPMD) simulations, discussed in the next paragraph. Moreover, a way to avoid even this arbitrariness has been recently proposed [60], replacing the short-range damping function by an estimation of the Pauli exchange repulsion given in terms of solely WFCs and spreads.

This method has been further improved [59] to minimize the computational cost. In this implementation a maximum deviation of the WFCs and atomic reference positions is used as a discriminating quantity for either the recalculation of the MLWF or their propagation as classic-like objects during the dynamics. Consequently, the update of MLWFs and hence the related recalculation of the $\mathrm{vdW} \mathrm{C}_{6}$ coefficients can be skipped for several thousands of simulation steps, leading to a remarkable decrease of the overhead. For the benchmark of the system [59], this overhead amounts to about $1 \%$ of the total computational cost. More precisely, the MLWF scheme for vdW inclusion framework in a DFT can be selected and applied according to the three possible computational schemes sketched in Fig. 4. 
- Method 1: Self-consistent time consuming but "exact" $\min \left\{E^{D F T, m}\right\} \Rightarrow \psi_{i} \Rightarrow w_{n}=U_{n}^{i} \psi_{i} \Rightarrow E^{D F T, m+1}=E^{D F T, m}+E_{v d W}^{W C}$
re-loop at each $t$

- Method 2: compute vdWC non self-consistently at each $t$ $\min \left\{E^{D F T}\right\}_{\forall t} \Rightarrow \psi_{i} \Rightarrow w_{n}=U_{n}^{i} \psi_{i} \Rightarrow E^{D F T}=E^{D F T}+E_{v d W}^{W C}$

- Method 3: if $\left|\mathbf{R}_{I}\left(t^{\prime}>t\right)-\mathbf{R}_{I}(\mathrm{t})\right|>$ tolerance, compute vdWC as above. Otherwise use the old $C_{n l}{ }^{6}$ and extrapolate WCs according to the movement of $\mathbf{R}_{I}\left(t^{\prime}>t\right)$

Fig. 4. Schemes for the implementation of three possible methods to add long-range van der Waals dispersion interactions to a standard density functional theory code.

The most appropriate choice depends on the specific system and its more or less complex chemical nature [67].

\section{First principles molecular dynamics}

All the quantum-mechanics based methods, including the HF and DFT ones, discussed so far, can be classified as (electro) static descriptions of the electronic and atomic structure. The atomic positions are generally either experimental coordinates from X-ray or neutron diffraction experiments or stationary points obtained via geometry optimization on reference configurations. Nonetheless, finite temperature and entropy effects play a major role in network forming materials. The method worldwide known as first principles molecular dynamics (FPMD) is the one that allowed this step forward in quantum simulations. As an extension of classical molecular dynamics (MD), instead of relying on some analytical ad-hoc analytical potential $V\left(\left\{\mathbf{R}_{I}\right\}\right)$, the interactions among atoms is directly computed from the DFT total energy $E^{\text {tot }}=E^{D F T}\left[\left\{\psi_{i}\right\},\left\{\mathbf{R}_{I}\right\}\right]$, which is simultaneously a function of the electronic structure and of the atomic coordinates. The interactions are assumed to be exclusively electrostatic, meaning that they depend just on the position and not on the velocities of the particles. From a historical standpoint, the first formulation of such a combined quantum (for the electrons) and classical (for the nuclei) was proposed by M. Born and J. R Oppenheimer in 1927 [72]. In this specific approach, nowadays known as the Born-Oppenheimer (BO) approximation, the ground state total energy is calculated (minimized) and subsequently used to compute gradients with respect to the nuclear position to propagate in time atoms in a Newton-like fashion, namely 


$$
\begin{gathered}
\frac{\delta E^{\mathrm{tot}}}{\delta \psi_{i}^{*}} \equiv H^{\mathrm{tot}} \psi_{i}(\mathbf{x})=\varepsilon_{i} \psi_{i}(\mathbf{x}) \\
M_{I} \ddot{\mathbf{R}}_{I}=-\nabla_{\mathbf{R}_{I}} \min _{\left\{\psi_{i}\right\}} E^{\mathrm{tot}}\left[\left\{\psi_{i}\right\},\left\{\mathbf{R}_{I}\right\}\right]
\end{gathered}
$$

The BO method requires the solution of Eq. (35) at each time step in the discretization of eq. (36), as done in any computer code. This is essential to ensure that electrons stay constantly on their ground state while the nuclei evolve dynamically. This feature is refereed to as "adiabatic approximation". For any further information we refer the reader to the rich literature $[9,17,25,26]$. A major leap forward in FPMD was the extended Lagrangean method proposed by R. Car and M. Parrinello in 1985 [73,74], in which the recalculation of the electronic structure at each step is avoided by including in the Lagrangean degrees of freedom the electronic wavefunctions. These become dynamical variables with their own equations of motion which, although representing a fictitious motion of the electronic structure, allow propagating the solution of the DFT KS orbitals instead of recalculating it at each movement of the nuclei. The original Car-Parrinello molecular dynamics (CPMD) Lagrangean reads

$$
\begin{aligned}
\mathrm{L}^{\mathrm{CP}}=\frac{1}{2} \sum_{I} M_{I} \dot{\mathbf{R}}_{I}^{2} & +\sum_{i} \mu \int d^{3} x\left|\dot{\psi}_{i}(\mathbf{x})\right|^{2}+\frac{1}{2} \sum_{\alpha} \eta_{\alpha} \dot{q}_{\alpha}^{2}-E^{t o t}\left[\rho,\left\{\mathbf{R}_{I}\right\}, q_{\alpha}\right] \\
& +\sum_{i j} \lambda_{i j}\left(\int d^{3} x \psi_{i}^{*}(\mathbf{x}) \psi_{j}(\mathbf{x})-\delta_{i j}\right)
\end{aligned}
$$

where the first three kinetic energies of the right-hand side refer to the nuclei, to the fictitious electronic degrees of freedom and any additional dynamical variable $q_{\alpha}(t)$ such as thermostats [75-77], barostats [78-80], reaction coordinates / collective variables $[81,82]$, etc. The fourth term is the potential energy as provided by DFT, whereas the final addendum is the constraint ensuring the orthonormality of the wavefunctions. The Euler-Lagrange equations of motion that this Lagrangean provides include then a coupling, via the DFT functional of all the dynamically evolving degrees of freedom and, explicitly, they read

$$
\begin{aligned}
\mu \ddot{\psi}_{i}(\mathbf{x}) & =-\frac{\delta E^{\mathrm{tot}}}{\delta \psi_{i}^{*}}+\sum_{j} \lambda_{i j} \psi_{j}(\mathbf{x}) \\
M_{I} \ddot{\mathbf{R}}_{I} & =-\nabla_{\mathbf{R}_{I}} E^{\mathrm{tot}} \\
\eta_{\alpha} \ddot{q}_{\alpha} & =-\frac{\partial E^{\mathrm{tot}}}{\partial q_{\alpha}}
\end{aligned}
$$

The fictitious electron mass $\mu$ written in front of the kinetic energy and corresponding equations of motion (38) regulates the rate at which the orbitals $\psi_{i}(\mathbf{x})$ are updated with 
respect to the dynamical evolution of the heavier nuclei. Hence, it is the parameter that controls the degree of adiabaticity all along the dynamics. A mathematical proof in the case of a finite Hilbert space can be found in the work of F. A. Bornemann and C. Schütte [83]. In that paper, the fundamental theorem shows that the CPMD trajectory $\left\{\mathbf{R}^{\mathrm{CP}}(t)\right\}$ evolves close to the truly adiabatic BO one $\left\{\mathbf{R}^{\mathrm{BO}}(t)\right\}$ and the upper bound is the square root of the electron mass $\mu$

$$
\left|\mathbf{R}^{C P}(t)-\mathbf{R}^{B O}(t)\right|<C \cdot \sqrt{\mu}
$$

where $\mathrm{C}$ is a positive constant. Empirical numerical verifications have been reported by the group of G. Galli $[84,85]$. The equations of motion are implemented in discrete finite differences [9]. Namely, second derivatives with respect to the time are written in the incremental form $d^{2} f(\mathrm{t}) / d t^{2} \sim[f(\mathrm{t}+\Delta t)+f(\mathrm{t}-\Delta t)-2 f(\mathrm{t})] / \Delta t^{2}$. Thus, the dynamical variables $\mathbf{R}_{I}(\mathrm{t})$ are updated at a rate $\Delta t$, while the electronic degrees of freedom are updated at a rate $\Delta t / \mu^{1 / 2}$. In fact, the discrete form that the equations of motion for the fictitious electronic degrees of freedom assume in a computer code read

$$
\begin{array}{r}
\frac{\mu}{\Delta t^{2}}\left[c_{i}(\mathbf{G}, t+\Delta t)+c_{i}(\mathbf{G}, t-\Delta t)-2 c_{i}(\mathbf{G}, t)\right]= \\
-\sum_{\mathbf{G}^{\prime}}\left\langle\mathbf{G}\left|H^{C P}\right| \mathbf{G}^{\prime}\right\rangle c_{i}\left(\mathbf{G}^{\prime}\right)+\sum_{j} \Lambda_{i j} c_{j}(\mathbf{G})
\end{array}
$$

Standard values for the time step and the fictitious electronic mass are generally 3-5 au and 300-600 au, respectively.

As a recent example in the field of chalcogenide glasses, this approach has allowed for the simulation of a realistic amorphous $\mathrm{GeS}_{2}$ surface [86] using a slab model composed of 480 atoms (Fig. 5). The interest in such a calculation stems from the fact that both structural and electronic properties of this amorphous chalcogenide surfaces make them promising candidates for applications in various fields [87]. In particular, the high polarizability of chalcogenide surfaces, as opposed to the majority of the oxide-based materials, finds practical applications in heterogeneous catalysis and gas phase separation. Hence, reliable FPMD simulations can become virtual experiments for the design of efficient processes and the testing of chalcogenide compounds with high specific surface area $\left(10-500 \mathrm{~m}^{2} / \mathrm{g}\right)$. 


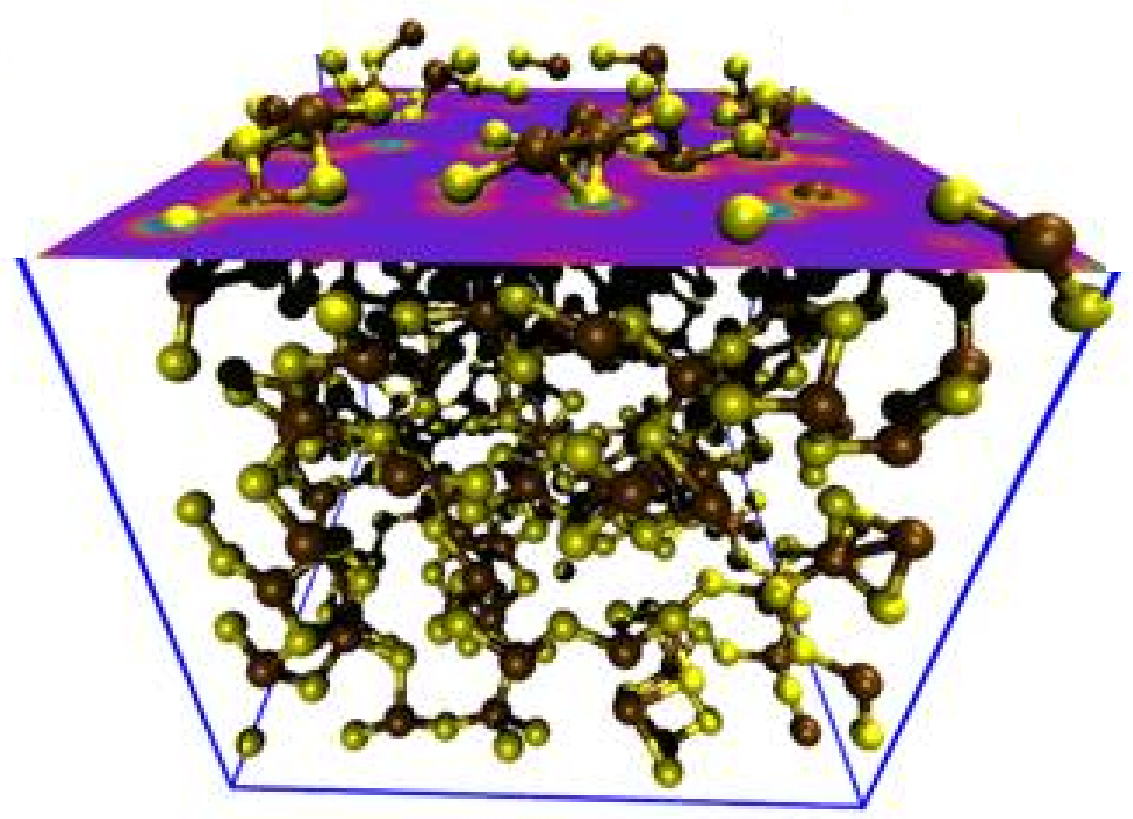

Fig. 5. Glassy $\mathrm{GeS}_{2}$ surface (480 atoms) with the local electrostatic potential shown as a density contour on a plane at about $2 \AA$ from the top. Colors code: Ge atoms, ochre; $\mathrm{S}$ atoms, yellow.

Analogously, and on the same line, porous glassy $\mathrm{GeS}_{2}$ have displayed characteristic pore widths of about $3.6 \mathrm{~nm}$. Such a peculiar distance is well fit to confine $\mathrm{CO}_{2}$ molecules at ambient conditions, namely $298 \mathrm{~K}$ and 1-10 bar. This confinement effect has been highlighted via dynamical atomic scale simulations [88-90] and an instructive snapshot of this confinement phenomenon is given in Fig. 6. Indeed, the adsorption and gas separation ability shown by amorphous porous chalcogenides such as $\mathrm{GeS}_{2}$ are attracting an increasing interest since they are promising systems for applications as gas sensors or as nanocatalysts for gas-phase reactions. Using a realistic molecular model of such amorphous adsorbents, it has been shown that they can be used efficiently to separate different gas-phase molecules $\left(\mathrm{H}_{2}, \mathrm{CO}_{2}, \mathrm{CH}_{4}, \mathrm{~N}_{2}\right)$ for environmental and energy storage applications [88]. In addition to shedding light on the microscopic adsorption mechanisms, previously escaping experimental probes, it has been found that co-adsorption in this class of porous materials can be described in terms of a simple thermodynamic model. Yet, to arrive at this general conclusion, accurate descriptions of the pores within the FPMD approach were deemed essential to realistically model the system. Hence, these results paved the way for the design of gas separation membranes using the large family of porous chalcogenides [89,90]. 


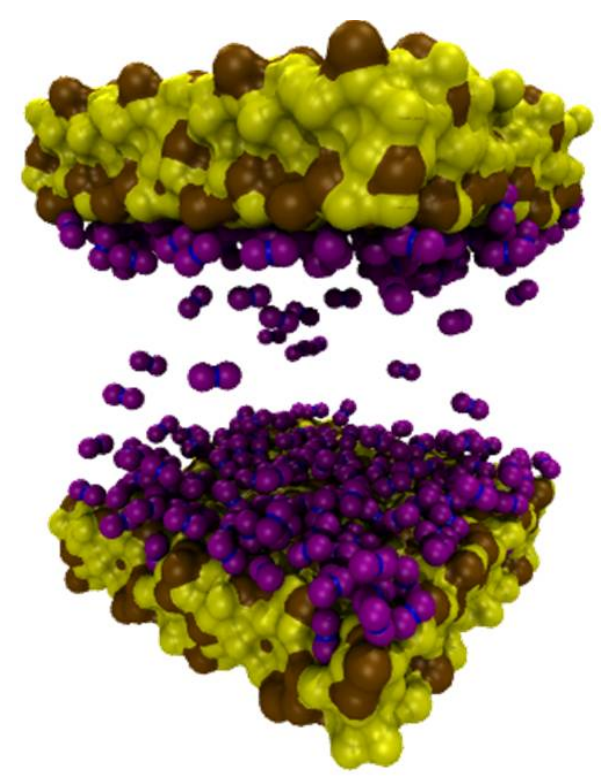

Fig. 6. Porous amorphous $\mathrm{GeS}_{2}$ (upper and lower yellow-brown slabs) confining $\mathrm{CO}_{2}$ molecules at ambient conditions. Colors code: $\mathrm{GeS}_{2}$ : $\mathrm{Ge}$ atoms, ochre and $\mathrm{S}$ atoms, yellow; $\mathrm{CO}_{2}$ : $\mathrm{C}$, blue and $\mathrm{O}$, purple.

The fact that the electronic structure is explicitly taken into account in FPMD and, specifically, in the CPMD approach, allows for a straightforward calculation of dielectric properties. Indeed, KS electron wavefunctions are updated - and available - at each step of the dynamics, and their Wannier unitary transformation make feasible the calculation of the instantaneous dipole moment on the fly with no appreciable computational burden. From the MLWF centers $\mathbf{r}_{\mathrm{n}}(t)=\left(x_{n}, y_{n}, z_{n}\right)$ and the atomic positions $\mathbf{R}_{I}(t)$ available at each time along the trajectory one can then obtain the total dipole moment of the system as a simple sum

$$
\mathbf{P}(t)=\mathbf{p}_{I}(t)+\mathbf{p}_{e}(t)=\sum_{I} Z_{I} \mathbf{R}_{I}(t)-\sum_{n} f_{n} \mathbf{r}_{\mathrm{n}}(t)
$$

where $Z_{I}$ is the valence charge of the $I$-th atom and $f_{n}$ the occupation number of the $n$-th Wannier orbital. It is then straightforward to compute the dipole-dipole autocorrelation function, and from this, upon a simple Fourier transform, the infra-red (IR) absorption coefficient of the material can be directly obtained [91-93] as

$$
\alpha(\omega)=\frac{4 \pi \omega \tanh (\beta \hbar \omega)}{3 \hbar n(\omega) \cdot c V} \int_{0}^{\infty}\langle\mathbf{P}(t) \cdot \mathbf{P}(0)\rangle \cdot e^{i \omega t} d t
$$

where $\beta=1 / k_{B} T$ depends on the temperature $T$ at which the simulation was conducted, and the rest are known constants (speed of light $c$, volume of the simulation cell $V$, etc.). This is basically the imaginary part of the refractive index $\varepsilon_{2}(\omega)=c \alpha(\omega) / 2 \omega$ and the 
real part $\varepsilon_{1}(\omega)$, i.e. the (real) refractive index of the material, can be computed from the standard Kramers-Kronig [94,95] relation

$$
\varepsilon_{1}(\omega)=1+\frac{2}{\pi} P \int_{0}^{\infty} \frac{\omega^{\prime} \cdot \alpha(\omega)}{\omega^{\prime 2}-\omega^{2}} d \omega^{\prime}
$$

This allows for a full characterization of the optical properties of a system which can be directly compared to the experimental measurements.

Such an approach has been successfully used, for instance, to investigate the dielectric and optical response of a series of $\mathrm{Ge}_{x} \mathrm{Se}_{1-x}$ compounds [96]. Long-lasting ( $\left.\sim 84 \mathrm{ps}\right)$ dynamical simulations in which the dipole moment was computed on the fly at each step could provide the adsorption coefficient of eq. (44) for a range of frequencies between 0.5 and $500 \mathrm{~cm}^{-1}$ (Fig.7, upper panel). Clearly, the total simulation time is crucial in getting accurate low frequencies $\left(1 \mathrm{~cm}^{-1} \sim 33.36 \mathrm{ps}\right)$, and this fixes the simulation time, i.e. computational workload required. Instead the high frequencies $\left(500 \mathrm{~cm}^{-1} \sim 0.07 \mathrm{ps}\right)$ are easily obtained within a few steps $(\sim 60)$ given the small $\Delta t$ $(0.10 \mathrm{fs})$ used in the numerical integration (eq. 42). The agreement with the experimental outcome [97] is appreciable (see Fig. 7).

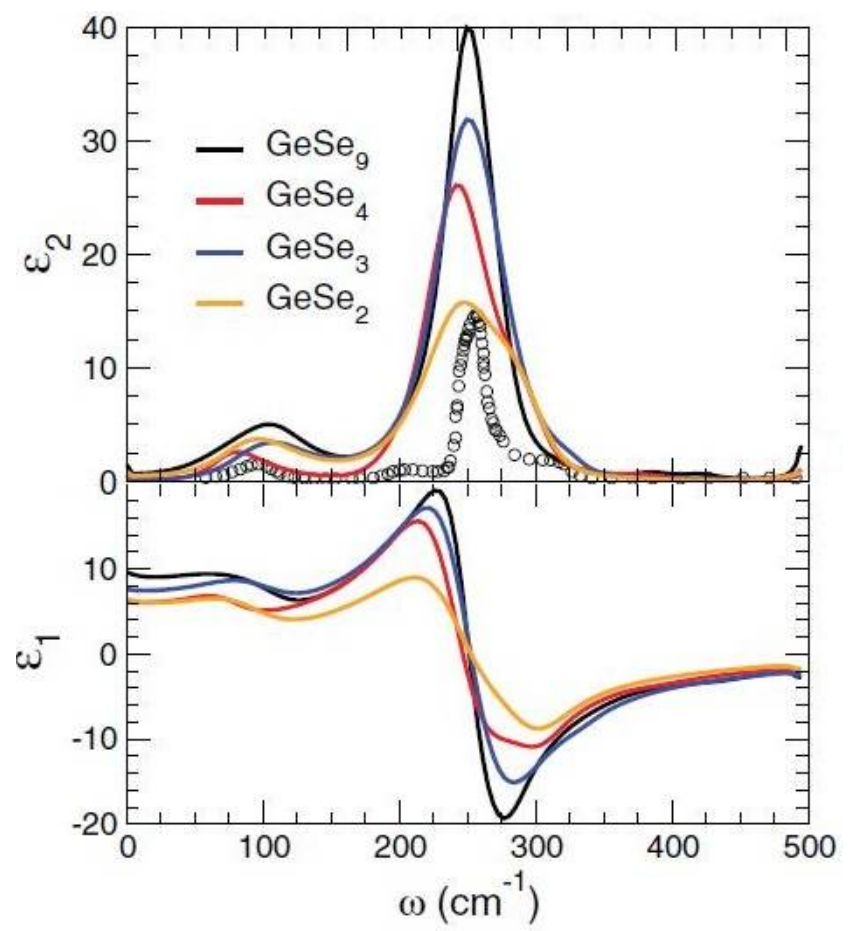

Fig. 7. Imaginary $\left(\varepsilon_{2}(\omega)\right)$ and real $\left(\varepsilon_{1}(\omega)\right)$ parts of the dielectric constant (color lines) as a function of the frequency for a series of simulated $\mathrm{Ge}_{x} \mathrm{Se}_{1-x}$ amorphous systems compared to experiments (circles). 
What are the progresses made so far to increase the computational reach of these approaches and allow for calculations on larger systems and extended temporal intervals? In what follows we provide some insights into these issues. In 2007, an efficient Car-Parrinello-like approach to the BO molecular dynamics has been proposed [98], making use of a predictor-corrector method for the integration of the electronic degrees of freedom. This has led to the so-called second-generation CPMD (SGCPMD). The details about the method have already been presented and discussed elsewhere $[17,74,98]$. For the sake of completeness, we limit here our review to the main differences with respect to a standard CPMD scheme. The basic idea is to exploit simultaneously the advantages of the BO and the CPMD methods. In a "nutshell", the first method makes use of first order equations of motion to minimize the electronic structure, while the second one propagates the electronic orbitals via second order equations of motion. The merging of these two methodologies leads to the following Langevin-like equations of motion for the electronic degrees of freedom

$$
\mu \frac{d^{2}}{d t^{2}}\left|\psi_{i}\right\rangle+\gamma \frac{d}{d t}\left|\psi_{i}\right\rangle=-\frac{\delta E_{N S C}}{\delta\left\langle\psi_{i}\right|}+\sum_{j} \Lambda_{i j}\left|\psi_{i}\right\rangle
$$

in which we can still recognize the CPMD-like part in the first term on the left-hand side, the BO-like one in the second one and then the familiar functional derivative of the total energy and the orthonormality constraint. The label NSC (non-self-consistent) on the energy functional is a reminder of the fact that a single BO step is done within this formalism, not the entire procedure typical of full re-optimization of the wavefunctions. This is kept under control with the additional parameter $\gamma$ and the fact that not the entire BO self-consistent procedure is implemented, but just a single iteration grants a computational workload close to a regular CPMD scheme. The associated equations of motion for the nuclei become then

$$
M_{I} \ddot{\mathbf{R}}_{I}=-\frac{\partial E_{N S C}}{\partial \mathbf{R}_{I}}+\sum_{i, j} \Lambda_{i j} \frac{\partial}{\partial \mathbf{R}_{I}}\left\langle\psi_{i} \mid \psi_{j}\right\rangle-2 \frac{\partial\left\langle\psi_{i}\right|}{\partial \mathbf{R}_{I}}\left(\frac{\delta E_{N S C}}{\delta\left\langle\psi_{i}\right|}-\sum_{j} \Lambda_{i j}\left|\psi_{j}\right\rangle\right)
$$

The somehow complicated second term, beside the calculation of the forces in terms of the gradient of the total energy (as usual) contains extra-components arising from the use of atom-centered localized basis functions, thus explicitly dependent on the atomic coordinates $\mathbf{R}_{I}$. The use of velocity-dependent (first order time derivatives) terms, 
although the wavefunctions are not self-consistently converged, introduces a friction term. This damps the orbitals close to the BO surface, thus allowing for larger integration steps with respect to the regular CPMD integration and resulting in an appreciable boost of the dynamical simulations.

In the field of network forming materials and, specifically, in phase change materials (PCMs) ternary compounds, the SGCPMD approach has been used to generate glassy $\mathrm{Ga}_{4} \mathrm{Sb}_{6} \mathrm{Te}_{3}$. This peculiar compound is one of the most promising materials for optical support and memory devices, since it features nearly all the properties sought, namely:

- High stability of amorphous and crystalline phase.

- Large contrast in optical properties and/or resistance change.

- Large cycle number of reversible transitions.

- High chemical stability.

- High laser light absorption

Yet, obtaining a reliable amorphous phase is extremely challenging. The standard simulation protocol, in these cases, is a melting of the crystal phase to a liquid state. Then, this liquid phase has to undergo a long equilibration to allow for a sufficient randomization of the atomic positions. Finally, a sufficiently low cooling process has to be simulated in order to allow the system to relax and to readjust the local bonding environment during the quench phase up to room temperature [99]. A schematic summary of the procedure adopted is reported in Fig. 8.

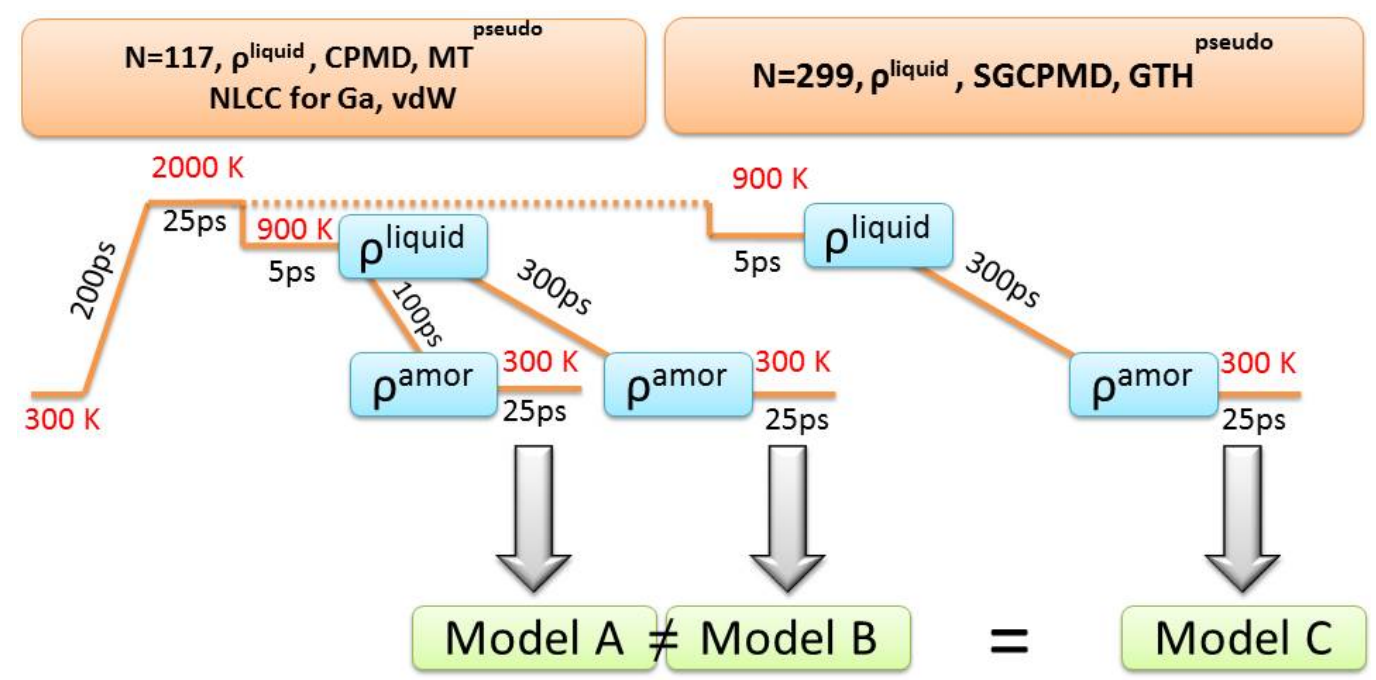

Fig. 8. Comparison of first principles molecular dynamics simulations within either the CPMD or the SGCPMD schemes adopted to generate a model system for the $\mathrm{Ga}_{4} \mathrm{Sb}_{6} \mathrm{Te}_{3}$ ternary phase-change material. 
As it can be seen, long cooling times ( $\sim 300 \mathrm{ps})$ were required to obtain a system, here indicated either as "Model B" (via regular CPMD simulations, 117 atoms) or "Model C" (larger system of 299 atoms by SGCPMD simulations).
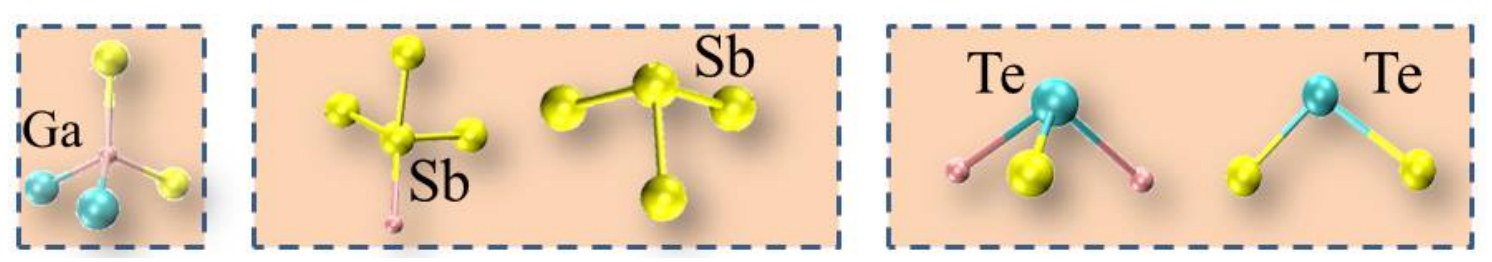

Fig. 9. Fundamental local structures constituting an amorphous PCM Ga $\mathrm{Sb}_{6} \mathrm{Te}_{3}$.

The obtained amorphous $\mathrm{Ga}_{4} \mathrm{Sb}_{6} \mathrm{Te}_{3}$ has been shown to contain the fundamental motifs shown in Fig. 9 and the material as a whole could be rationalized [99] into a superstructure composed of two subnetworks, one being a $(\mathrm{GaTe})_{3}$, and a second one being a $\mathrm{GaSb}_{6}$, this, in practice, $\mathrm{Ga}_{4} \mathrm{Sb}_{6} \mathrm{Te}_{3} \equiv\left((\mathrm{GaTe})_{3}-\mathrm{GaSb}_{6}\right)$.

\section{Final remarks}

The overview presented here about first-principle based methods is specifically oriented to amorphous chalcogenide materials. We aimed at offering to the reader a collection of advanced computational methods and techniques, supported by a few selected examples that we consider as representative or pioneering in the field. Modern algorithms and extension to machine-learning protocols are highlighted as forefront developments of either well-assessed "traditional" or novel first-principles based techniques nowadays routinely used in the simulation of disordered condensed phases.

For the sake of completeness, we stress that many more developments and applications are available in the rich literature, part of which is reported in the included list of references, and that could offer a wider panorama to researchers interested or working in the field.

\section{Acknowledgements}

M.B. thanks Pôle HPC, Equipex Equip@Meso / CPER Alsacalcul at the University of Strasbourg, and Grand Equipement National de Calcul Intensif (GENCI) under allocation DARI-A2 A0060906092. G.O. acknowledges the Fédération de Recherche en Matériaux et Nanosciences Region Grand-Est (project HARWEST) and the Seed Money program of Eucor - The European Campus (project MEDIA) for financial support. 


\section{References}

1. E.D. Zanotto, J.C. Mauro J. Non-Cryst. Solids 471 (2017) 590. Doi: 10.1016/j.jnoncrysol.2017.05.019

2. B.J. Alder, T.E.J. Wainwright, Chem. Phys. 27 (1957) 1208. doi:10.1063/1.1743957

3. B.J. Alder, T.E.J. Wainwright, J. Chem. Phys. 31 (1959) 459. Doi: 10.1063/1.1730376

4. A. Rahman, Phys. Rev. A 136 (1964) A405. Doi: 10.1103/PhysRev.136.A405

5. F.H. Stillinger, A. Rahman, J. Chem. Phys. 60 (1974) 1545. Doi: 10.1063/1.1681229

6. R. McWeeny, Methods of Molecular Quantum Mechanics, Academic Press, London, UK, 1992.

7. I.N. Levine, Quantum Chemistry, Allyn and Bacon Ed., Boston, USA, 1983.

8. A. Szabo, N.S. Ostlund, Modern Quantum Chemistry - Introduction to Advanced Electronic Structure Theory, McGraw-Hill Publishing Co., New York, USA, 1989.

9. D. Marx, J. Hutter, Ab initio molecular dynamics: Basic Theory and Advanced Methods, Cambridge University Press, New York, USA, 2009.

10. T. Pang, An Introduction to Computational Physics, Cambridge University Press, Cambridge, UK, 1997.

11. A. Edwards, W. Shedd, R. Pugh, Hartree Fock and DFT Study of Models for Amorphous Chalcogenide Alloys, American Physical Society, Annual March Meeting, March 12-16, 2001 Washington State Convention Center Seattle, Washington Meeting ID: MAR01, abstract \#S14.010

12. M. Rekhis, O. Ouamerali, L. Joubert, V. Tognetti, C. Adamo, J. Mol. Struct. THEOCHEM 863 (2008) 79. Doi: 10.1016/j.theochem.2008.05.018

13. A. Fernando, K.L. Dimuthu, M. Weerawardene, N.V. Kaimova, C.M. Alkens, Chem. Rev. 115 (2015) 6112. Doi: 10.1021/cr500506r

14. C. Møller, M.S. Plesset, Phys. Rev. 46 (1934) 618. doi: 10.1103/PhysRev.46.618

15. J. Jellinek, V. Bonačić-Koutecký, P. Fantucci, M.J. Wiechert, J. Chem. Phys. 101, (1994) 10092. Doi: 10.1063/1.467997

16. M. Kobayashi, Y. Imamura, Y., H. Nakai, J. Chem. Phys. 127 (2007) 074103. doi: $10.1063 / 1.2761878$

17. M. Boero, A. Bouzid, S. Le Roux, B. Ozdamar, C. Massobrio, First-Principles Molecular Dynamics Methods: An Overview, in Frontiers and challenges in Molecular Dynamics Simulations of Disordered Materials: From network glasses to phase change memory alloys, pag. 35-55, Springer, Berlin Heidelberg, Germany, 2015. ISBN: 978-3-319-15674-3

18. R. Fairman, B. Ushkov, B., Semiconducting Chalcogenide Glass II: Properties of 
Chalcogenide Glasses, Semiconductors and Semimetals vol. 79, ed. by R.K. Willardson, E.R. Weber, Elsevier, San Diego, USA, 2004.

19. P. Hohenberg, W. Kohn, W. Phys. Rev. 136 (1964) B864. Doi: 10.1103/PhysRev.136.B864

20. W. Kohn, L.J. Sham, Phys. Rev. 140 (1965) A1133. Doi: 10.1103/PhysRev.140.A1133

21. D.J. Defeers, B.A. Levi, S.K. Pollack, W.J. Hehre, J.S. Binkley, J. S., J.A. Pople, J. Am. Chem. Soc. 101, (1979) 4085. Doi: 10.1021/ja00509a013

22. J.A. Pople, M. Head-Gordon, D.J. Fox, K. Raghavachari, L.A. Curtiss, J. Chem. Phys. 90 (1989) 5622. Doi: 10.1063/1.456415

23. J.A. Pople, H.B. Schlegel, R. Krishnan, D.J. Defrees, J.S. Binkley, M.J. Frisch, R.A. Whiteside, R.F. Hout, W.J. Hehre, Int. J. Quantum Chem. Symp. 15 (1981) 269. Doi: 10.1002/qua.560200829

24. B.G. Johnson, P.M.W. Gill, J.A. Pople, J. Chem. Phys. 98 (1993) 5612-5626. Doi: $10.1063 / 1.464906$

25. R.G. Parr, W. Yang, Density-Functional Theory of Atoms and Molecules, Oxford University Press, New York, USA, 1989.

26. M. Boero, M. Tateno, Quantum Theoretical Approaches to Proteins and Nucleic Acids, in The Oxford Handbook of Nanoscience and Technology Volume 1, Chapter 17, pag. 549, Oxford University Press, UK, 2010. ISBN: 978-0-19-9533040-6

27. A.D. Becke, Phys. Rev. A 38 (1988) 3098. Doi: 10.1103/PysRevA.38.3098

28. A.D. Becke, J. Chem. Phys. 96 (1992) 2155. Doi: 10.1063/1.462066

29. A.D. Becke, J. Chem. Phys. 98 (1983) 5648-5652. Doi: 10.1063/1.464913

30. J.P. Perdew, A. Zunger, Phys. Rev. B 23 (1982) 5048. Doi: 10.1103/PhysRevB.23.5048

31. J.P. Perdew, Phys. Rev. B 33 (1986) 8822. Doi: 10.1103/PhysRevB.33.8822

32. J.P. Perdew, Y. Wang, Phys. Rev. B 45 (1992) 13244. Doi: 10.1103/PhysRevB.45.13244

33. J.P. Perdew, J.A. Chevary, S.H. Vosko, K.A. Jackson, M.R. Pederson, D.J. Singh, C. Fiolhais, Phys. Rev. B 46 (1992) 6671. Doi: 10.1103/PhysRevB.46.6671

34. J.P. Perdew, K. Burke, M. Ernzerhof, Phys. Rev. Lett. 77 (1996) 3865-3868. Doi: 10.1103/PhysRevLett.77.3865

35. S.H. Vosko, L. Wilk, M. Nusair Can. J. Phys. 58 (1980) 1200. doi: 10.1139/p80-159

36. C. Lee, W. Yang, R.G. Parr, Phys. Rev. B 37 (1988) 785. Doi: 10.1103/PhysRevB.37.785

37. F.A. Hamprecht, A.J. Cohen, D.J. Tozer, N.C. Handy, J. Chem. Phys. 109 (1998) 
6264-6271. Doi: 10.1063/1.477267

38. N.C. Handy, A.J. Cohen, J. Chem. Phys. 116 (2002) 5411. Doi: 10.1063/1.1457432

39. A. Pedersen, L. Pizzagalli, H. Jónsson, New J. Phys. 19 (2017) )063018. Doi: 10.1088/1367-2630/aa732e

40. G. Ori, C. Massobrio, A. Bouzid, M. Boero, B. Coasne, Phys. Rev. B 90 (2014) 045423. doi: 10.1103/PhysRevB.90. 045423.

41. G.C. Sosso, G. Miceli, S. Caravati, J. Behler, M. Bernasconi, Phys. Rev. B 85 (2012) 174103. Doi: 10.1103/PhysRevB.85.174103

42. V.L. Deringer, N. Bernstein, A.P. Bartók, M.J. Cliffe, R.N. Kerber, L.E. Marbella, C.P. Grey, S.R. Elliott, G. Csáni, J. Phys. Chem. Lett. 9 (2018) 2879. Doi: 10.1021/acs.jpclett.8b00902

43. Y. Huang, J. Kang, W.A. Goddard III, L.-W. Wang, Phys. Rev. B 99 (2019) 064103, Doi: 10.1103/PhysRevB.99.064103

44. J. Behler, M. Parrinello, Phys. Rev. Lett. 98 (2007) 146401. Doi: 10.1103/PhysRevLett.98.146401

45. S. Gabardi, E. Baldi, E. Bossoni, D. Campi, S. Caravati, G.C. Sosso, J. Behler, M. Bernasconi, J. Hys. Chem. C 121 (2017) 23827. Doi: 10.1021/acs.jpcc.7b09862

46. F.C. Mocanu, K. Konstantinou, T.H. Lee, N. Bernstein, V.L. Deringer, G. Csányi, S.R. Elliott J. Phys. Chem. B 122 (2018) 8998. Doi: 10.1021/acs.jpcb.8b06476

47. W. Zhang, R. Mazzarello, M. Wuttig, E. Ma Nat. Rev. Mater. 4 (2019) 150. Doi: 10.1038/ s41578-018-0076-X

48. P.J. Stephens, F.J. Devlin, C.F. Chabalowski, C. F., M.J. Frisch, J. Phys. Chem. 98 (1994) 11623. Doi: 10.1021/j100096a001

49. J. Heyd, G.E. Scuseria, M. Ernzerhof, J. Chem. Phys. 118 (2003) 8207. Doi: $10.1063 / 1.15644060$

50. J.P. Perdew, M. Ernzerhof, K. Burke, J. Chem. Phys. 105 (1996) 9982. Doi: $10.1063 / 1.472933$

51. T. Kaewmaraya, M. Ramzan, H. Löfas, R. Ahuja, J. Appl. Phys. 113 (2013) 033510. Doi: $10.1063 / 1.4775715$

52. S. Grimme, J. Comput. Chem. 27 (2006) 1787. Doi: 10.1002/jcc.20495

53. T. Schwabe, S. Grimme, Phys. Chem. Chem. Phys. 9 (2007) 3397. Doi: 10.1039/B704725H

54. D.C. Langreth, M. Dion, H. Rydberg, E. Schröder, P. Hyldgaard, B.I Lundqvist, Int. J. Quantum Chem. 101 (2005) 599. Doi: 10.1002/qua.20315

55. O.A. Vydrov, T. Van Voorhis, Phys. Rev. Lett. 103 (2009) 063004. Doi: 10.1103/PhysRevlett.103.063004 
56. M. Obata, M. Nakamura, I. Hamada, T. Oda, J. Phys. Soc. Japan 84 (2015) 024715. Doi: 10.7566/JPSJ.84. 024715

57. P.L. Silvestrelli, Phys. Rev. Lett. 100 (2008) 053002. Doi:

10.1103/PhysRevLett.100.053002

58. P.L. Silvestrelli, J. Phys. Chem. A 113 (2009) 5224. Doi: 10.1021/jp811138n

59. T. Ikeda, M. Boero, J. Chem. Phys. 143 (2015) 194510. Doi: 10.1063/1.4935932

60. P.L. Silvestrelli, A. Ambrosetti, J. Chem. Phys. 150 (2019) 164109. Doi: $10.1063 / 1.5093125$

61. A. Bouzid, C. Massobrio, M. Boero, G. Ori, K. Sykina, E. Furet, Phys. Rev. B 92 (2015) 134208. Doi: 10.1103/PhysRevB.92.134208

62. Z. Chaker, G. Ori, C. Tugène, S. Le Roux, M. Boero, C. Massobrio, E. Martin, A. Bouzid, J. Non-Cryst. Solids $498 \quad$ (2018) 167. Doi: 10.1016/j.jnoncrysol.2018.06.031

63. E. Lampin, A. Bouzid, G. Ori, M. Boero, C. Massobrio, J. Chem. Phys. 147 (2017) 044504. Doi: 10.1063/1.4986166

64. G.H. Wannier, Phys. Rev. 52 (1937) 191. Doi: 10.1103/PhysRev.51.191

65. N. Marzari, D. Vanderbilt, Phys. Rev. B 56 (1997) 12847. Doi: 10.1103/PhysRevB.56.12847

66. R. Resta, Phys. Rev. Lett. 80 (1988) 1800. Doi: 10.1103/PhysRevLett.80.1800

67. C. Massobrio, E. Martin, Z. Chaker, M. Boero, A. Bouzid, S. Le Roux, G. Ori, Front. Mater. 5 (2018) 78. Doi: 10.3389/fmats.2018.00078

68. S. Le Roux, A. Bouzid, M. Boero, C. Massobrio, Phys. Rev. B 86 (2012) 224201. Doi : 10.1103/hysRevB.86. 224201

69. M. Bauchy, M. Micoulaut, M. Boero, C. Massobrio, Phys. Rev. Lett. 110 (2013) 165501. Doi: 10.1103/PhysRevLett.110. 165501

70. M. Celino, S. Le Roux, G. Ori, B. Coasne, A. Bouzid, M. Boero, C. Massobrio, Phys. Rev. B 88 (2013) 174201. Doi: 10.1103/hysRevB.88.174201

71. A. Bouzid, G. Ori, M. Boero, E. Lampin, C. Massobrio, Phys. Rev. B 96 (2017) 224204. Doi: 10.1103/PhysRevB.96.224204

72. M. Born, J.R. Oppenheimer J. R. Annalen der Physik 84 (1927) 457. Doi: 10.1002/andp.19273892002

73. R. Car, M. Parrinello, Phys. Rev. Lett. 55, (1985) 2471. Doi: 10.1103/PhysRevLett.55.2471

74. M. Boero, A. Oshiyama, Car-Parrinello Molecular Dynamics in Encyclopedia of Nanotechnology, pag. 1-10, Springer, Berlin Heidelberg, Germany, 2015. Doi: 10.1007/978-94-007-6178-0_100946-1 
75. S. Nosé, Mol. Phys. 52 (1984) 255. Doi: 10.1080/00268978400101201

76. S. Nosé, J. Chem. Phys. 81 (1984) 511. Doi : 10.1063/1.447334

77. W.G. Hoover, Phys. Rev. A 31 (1985) 1695. Doi: 10.1103/PhysRevA.31.1695

78. H.C. Andersen, J. Chem. Phys. 72 (1980) 2384. Doi: 10.1063/1.439486

79. M. Parrinello, A. Rahman, Phys. Rev. Lett. 45 (1980) 1196. Doi: 10.1103/PhysRevLett.45.1196

80. M. Parrinello, A Rahman, J. Appl. Phys. 52 (1981) 7182. Doi: 10.1063/1.328693

81. M. Iannuzzi, A. Laio, M. Parrinello, Phys. Rev. Lett. 90 (2003) 238302. Doi: 10.1103/PhysRevLett.90.238302

82. M. Boero, T. Ikeshoji, C.C. Liew, K. Terakura, M. Parrinello, J. Am. Chem. Soc. 2004 (126) 6280. Doi: 10.1021/ja049363f

83. F.A. Bornemann, C. Schütte, Numerische Matematik 78 (1998) 359. Doi: $10.1007 / \mathrm{s} 002110050316$

84. J.C. Grossman, E. Schwegler, E.W. Draeger, F. Gygi, G. Galli, J. Chem. Phys. 120 (2004) 300. Doi: 10.1063/1.1630560

85. E. Schwegler, J.C. Grossman, F. Gygi, G. Galli, J. Chem. Phys. 121 (2004) 5400. Doi: $10.1063 / 1.1782074$

86. G. Ori, C. Massobrio, A. Bouzid, M. Boero, B. Coasne, Phys. Rev. B 90 (2014) 045423. Doi: 10.1103/PhysRevB.90. 045423

87. T. Baba, Y. Kawamura Y Front. Energy Res. 4 (2016) 22. Doi: 10.3389/fenrg.2016.00022

88. G. Ori, C. Massobrio, A. Pradel, M. Ribes, B. Coasne, Langmuir 31 (2015) 6742. doi: 10.1021/acs.langmuir.5b00982

89. G. Ori, C. Massobrio, A. Pradel, M. Ribes, B. Coasne, Phys. Chem. Chem. Phys. 18 (2016) 13449. doi: 10.1039/C6CP00467A

90. Z. Chaker, A. Bouzid, B. Coasne, C. Massobrio, M. Boero, G. Ori, J. Non-Cryst. Solids 498 (2018) 288. Doi: 10.1016/j.jnoncrysol.2018.06.031

91. P.L. Silvestrelli, M. Bernasconi, M. Parrinello, Chem. Phys. Lett. 277 (1997) 478. doi: 10.1016/S0009-2614(97)00930-5

92. M. Boero, K. Terakura, T. Ikeshoji, C.C. Liew, M. Parrinello, J. Chem. Phys. 115 (2001) 2219. Doi: 10.1063/1.1379767

93. D.A. Schmidt, R. Scipioni, M. Boero, J. Phys. Chem. A 113 (2009) 7725-7729. Doi: 10.1021/jp9016932

94. H.A. Kramers, Transactions of Volta Centenary Congress 2 (1927) 545.

95. R. de L. Kronig, J. Opt. Soc. Am. 12, (1927) 547. Doi: 10.1364/JOSA.12.000547

96. M. Micoulaut. A. Kachmar, M. Bauchy, S. Le Roux, C. Massobrio, M. Boero, Phys. 
Rev. B 88 (2013) 054203. Doi: 10.1103/PhysRevB.88. 054203

97. G. Lukovsky, R.J. Nemanich, S.A. Solin, R.C. Keezer, Solid State Commun. 17, (1975) 1567. Doi: 10.1016/0038-1098(75)90997-7

98. T.D. Kühne, M. Krack, F.R. Mohamed, M. Parrinello, Phys. Rev. Lett. 98 (2007) 066401. doi: 10.1103/PhysRevLett.98.066401

99. A. Bouzid, S. Gabardi, C. Massobrio, M. Boero, M. Bernasconi, Phys. Rev. B 91 (2015) 184201. Doi: 10.1103/PhysRevB.91. 184201

\section{Abbreviation list}

HPC High-performance computing

MD Molecular Dynamics

HF Hartree-Fock

GVB Generalized valence bond

DFT Density functional theory

CI Configuration interaction

CASSCF Complete active space self consistent field

MP2 Second-order Møller-Plesset

KS Kohn-Sham

LDA Local density approximation

$\mathrm{XC}$ Exchange-correlation

GGA Generalized gradient approximation

PBE Perdew-Burke-Enrnzerhof

BLYP Becke-Lee-Yang-Parr

ML Machine learning

FF Force field

PES Potential energy surface

NN Neural network

B3LYP Three-parameter Becke-Lee-Yang-Parr

HSE Heyd-Scuseria-Ernzerhof

PBE0 Perdew-Burke-Ernzerhof with Hartree-Fock exchange

vdW van der Waals

MLWF Maximally localized Wannier functions

WFC Wannier function center 
FPMD First principles molecular dynamics

BO Born-Oppenheimer

CPMD Car-Parrinello molecular dynamics

SGCPMD Second-generation Car-Parrinello molecular dynamics

\section{Graphical Abstract:}

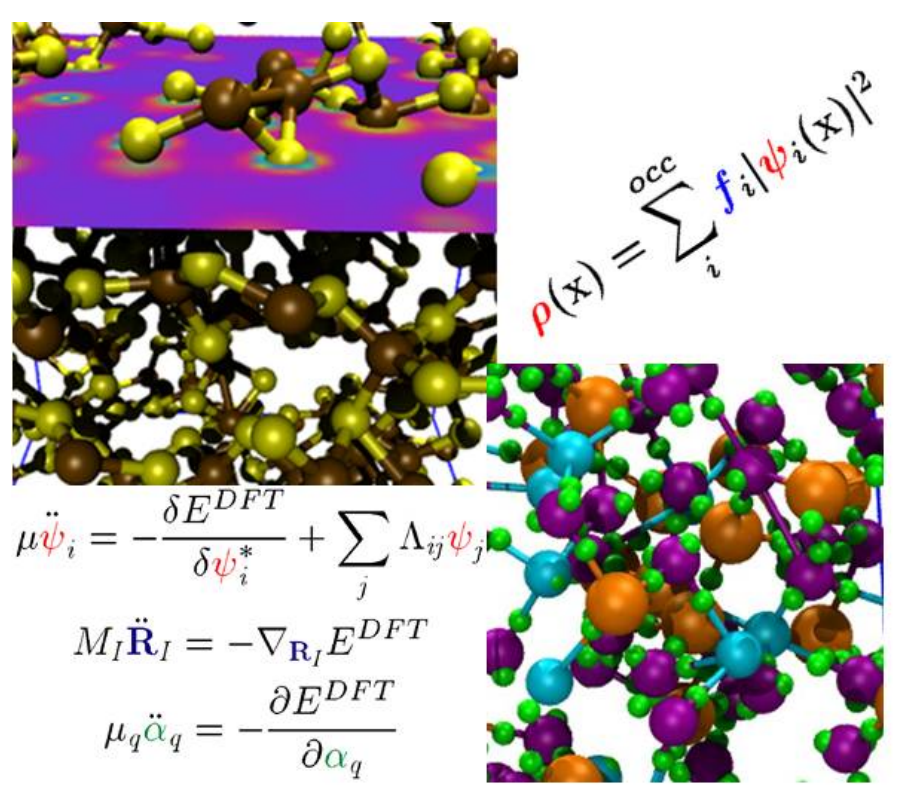




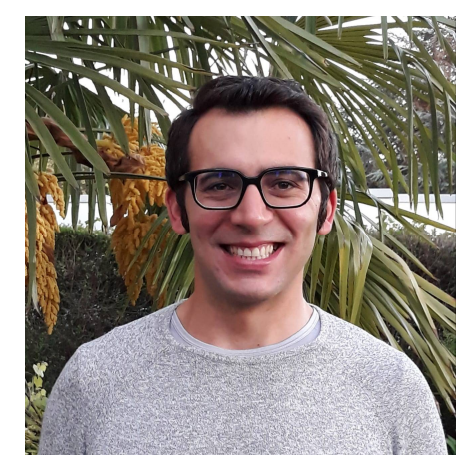

Guido Ori is a CNRS researcher at the Institut de Physique et Chimie des Matériaux of Strasbourg, France. He received his $\mathrm{PhD}$ in 2010 in Chemistry at the University of Modena with a thesis on multiscale modelling. He has a multidisciplinary interest from materials science to physical chemistry and is author of more than 30 publications in peer reviewed journals.

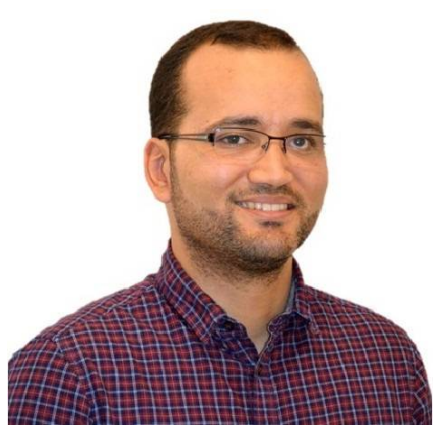

Assil Bouzid is CNRS researcher at the Institut de Recherche sur les Céramiques, France. He received his $\mathrm{PhD}$ in 2014 in Physics and Chemical-Physics at the University of Strasbourg. He has a consolidated experience in first principles simulations and in computer modeling for condensed matter physics. He is author of more than 30 publications in peer reviewed journals.

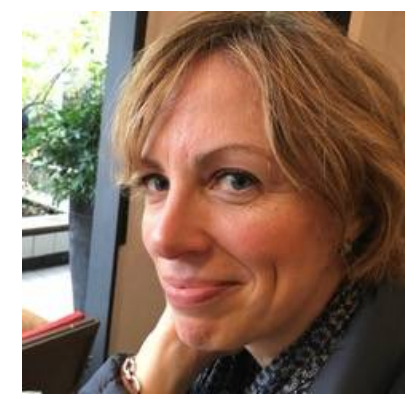

Evelyne Martin (also known as Lampin) is a CNRS Research Director at the Institute of Electronics, Microelectronics and Nanotechnology in Lille, France. She got her PhD in 1997 in materials science at University of Science and Technology in Lille. Expert in computational material science focused on thermal transport in nanomaterials, she is author of more than 50 publications in peer reviewed journals.

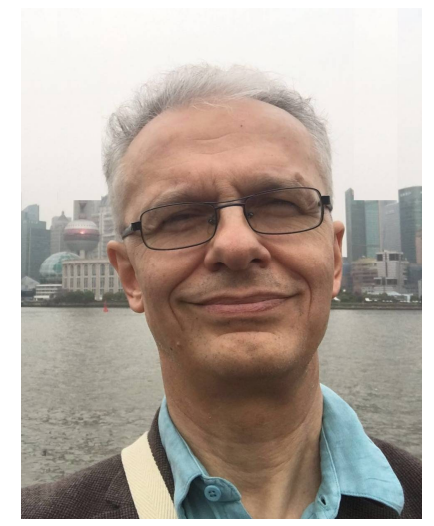

Carlo Massobrio is CNRS Senior Research Director and member of the CNRS Material Science board. He is a recognized computational material scientist in the area of first-principles molecular dynamics (FPMD) of disordered materials, organic -inorganic compounds and nanostructures. $\mathrm{He}$ has pioneered applications of FPMD to glassy chalcogenides, including a thorough analysis of exchange-correlation functionals. He is author of more than 180 publications in peer reviewed journals. 


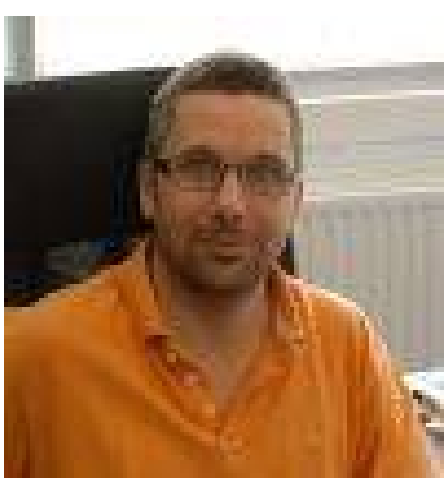

Sebastien Le Roux is research engineer at the Institut de Physique et Chimie des Matériaux of Strasbourg, France. He received his $\mathrm{PhD}$ in chemistry and physical chemistry of materials at 2008 at the University of Montpellier. He is expert in atomistic simulations, software developer (ISAACS, RINGS, RAD) for data post-processing in atomic-scale simulations and author of more than 30 peer reviewed publications.

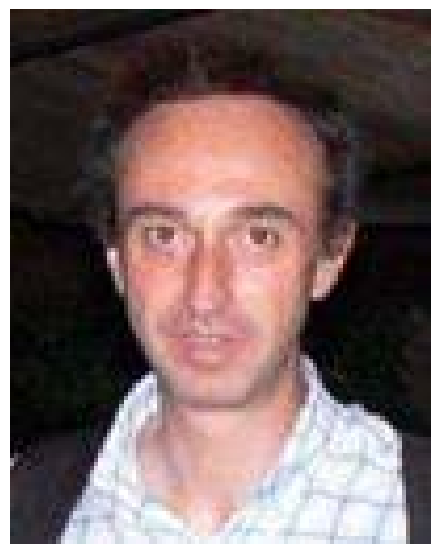

Mauro Boero is research director at the Institut de Physique et Chimie des Matériaux of Strasbourg, France, scientific director of the HPC Center of the University of Strasbourg and visiting full professor at Nagoya University, Japan. He received his $\mathrm{PhD}$ in 1994 in physics at the University of Turin. Expert in multi-scale modeling and co-developer of worldwide used HPC codes, including CPMD (www.cpmd.org). He is author of more than 170 articles in peer reviewed journals, 